\title{
Hadronic top-quark pair production in association with a hard jet at next-to-leading order QCD: phenomenological studies for the Tevatron and the $\mathrm{LHC}$
}

\author{
S. Dittmaier ${ }^{1}$, P. Uwer ${ }^{2}$, S. Weinzierl ${ }^{3, a}$ \\ ${ }^{1}$ Max-Planck-Institut für Physik (Werner-Heisenberg-Institut), 80805 München, Germany \\ ${ }^{2}$ Institut für Theoretische Teilchenphysik, Universität Karlsruhe, 76128 Karlsruhe, Germany \\ ${ }^{3}$ Institut für Physik, Universität Mainz, 55099 Mainz, Germany
}

Received: 14 October 2008 / Revised: 21 November 2008 / Published online: 20 December 2008

(C) Springer-Verlag / Società Italiana di Fisica 2008

\begin{abstract}
We report on the calculation of the next-toleading order QCD corrections to the production of topantitop-quark pairs in association with a hard jet at the Tevatron and at the LHC. Results for integrated and differential cross sections are presented. We find a significant reduction of the scale dependence. In most cases the corrections are below $20 \%$ indicating that the perturbative expansion is well under control. Moreover, the forward-backward charge asymmetry of the top quark, which is analyzed at the Tevatron, is studied at next-to-leading order. We find large corrections, suggesting that the definition of the observable has to be refined.
\end{abstract}

\section{Introduction}

The top quark is by far the heaviest elementary fermion in the Standard Model (SM). With a mass of $(172.6 \pm 1.4) \mathrm{GeV}$ [1] its mass is about 36 times larger than the mass of the next heaviest fermion, the bottom quark. The large mass has lead to various speculations on whether the top quark behaves as a normal quark or whether it plays a special role in particle physics. The electroweak $\mathrm{SU}(2) \times \mathrm{U}(1)$ gauge structure of the SM, which is successful in describing a large variety of measurements, would require the quark masses to be zero if the symmetry was unbroken. With the largest mass amongst the quarks it is thus natural to assume that the top quark is most sensitive to the mechanism of electroweak symmetry breaking. In particular, the fact that the top-quark mass is close to the scale of electroweak symmetry breaking-or equivalently, that the Yukawa coupling to the Higgs boson is very close to 1 -has motivated different scenarios in which

\footnotetext{
a e-mail: stefanw@thep.physik.uni-mainz.de
}

the top quark drives the electroweak symmetry breaking. More details can be found in recent review articles [2,3].

Ignoring the SM as the theory of particle physics one might still wonder whether the top quark, which is almost as heavy as a gold atom, behaves as a point-like particle. A deviation from the point-like nature would appear as anomalous moments yielding differential distributions different from the point-like case. Anomalous couplings to the gluon are most naturally probed via the production of an additional jet. An indirect measurement through the measurement of the total cross section is in general more difficult. This is in particular true when the interference term with the corresponding Born amplitude gives no contribution to the total cross section due to discrete symmetries.

In the context of the SM we have the remarkable fact that the electroweak top-quark interactions are completely determined through the aforementioned $\mathrm{SU}(2) \times \mathrm{U}(1)$ gauge structure of the SM. The only free parameter appearing in top-quark physics is thus the top-quark mass or equivalently the Yukawa coupling to the Higgs boson. Once this parameter is measured all remaining properties are predicted.

An important task for the ongoing Tevatron collider and the recently started LHC is the precise measurement of the top-quark properties. The ultimate goal is to measure the spin and the quantum numbers of the top quark as precisely as possible. Any deviation from the SM would signal new physics. There is a variety of measurements which are currently done at the Tevatron and will be continued at the LHC. The total cross section, which is currently measured at the Tevatron with an accuracy of about $10 \%$ [4], is expected to be measured at the LHC with an accuracy of 5\%. This measurement allows one to make precise tests of the production mechanism. Other important measurements comprise the cross section for single top-quark production [5-11], the W-polarization in top-quark decay or the spin correlations 
of top-quark pairs [12-20]. Of great interest is also the electric charge of the top quark and its couplings to the Z-boson and the Higgs boson. They can be constrained via measurements of the cross sections for $\mathrm{t} \overline{\mathrm{t}} \gamma, \mathrm{t} \overline{\mathrm{t}} Z$ [21], and $\mathrm{t} \overline{\mathrm{t}} H$ [22-25] production.

The production of a top-quark pair together with an additional jet is a further important reaction. This becomes already clear from the simple observation that a substantial number of events in the inclusive top-quark sample is accompanied by an additional jet. Depending on the energy of the additional jet the fraction of events with an additional jet can easily be of the order of $10-30 \%$ or even more. For example, at the LHC we find a cross section of $376 \mathrm{pb}$ for the production of a top-antitop-quark pair with an additional jet with a transverse momentum above $50 \mathrm{GeV}$. This is almost half of the total top-quark pair cross section which is 806 $\mathrm{pb}[26]$ if evaluated in next-to-leading order (NLO). ${ }^{1}$ For a more precise understanding of the topology of top-quark events it is thus important to have also an improved understanding of top-quark pair production together with a jet. As mentioned already above, this reaction provides a sensitive tool to search for anomalous top-quark-gluon couplings. The emission of an additional gluon also leads to a rather interesting property of the cross section: the differential cross section contains contributions from the interference of Codd and C-even parts of the amplitude [27-30], where C denotes the charge conjugation (for a similar effect in QED see for example [31, 32]). While for the total cross section these contributions cancel when integrating over the (symmetric) phase space, they can lead to a forward-backward charge asymmetry of the top quark, which is measured at the Tevatron $[33,34]$. It should be stressed that no parityviolating interactions are involved. Note that the naively defined forward-backward charge asymmetry is zero at the LHC due to the symmetric initial state. A definition that leads to a non-trivial prediction here requires one to select a preferred axis for each event [35], but it is not yet clear whether an asymmetry survives that is significant over all uncertainties. In inclusive top-quark pair production at the Tevatron the charge asymmetry appears first at 1 loop, because it results from interferences of C-odd with $\mathrm{C}$-even parts of double-gluon exchange between initial and final states. The asymmetry for the inclusive sample has been studied in detail in [27-30]. The available predictions for $\mathrm{t} \overline{\mathrm{t}}$ production - although of 1-loop order-describes this asymmetry only at leading-order (LO) accuracy in QCD. Recently the analysis has been extended to take large threshold logarithms at the next-to-leading-log (NLL) level into

\footnotetext{
${ }^{1}$ Both numbers correspond to a top mass of $174 \mathrm{GeV}$. The number for top-antitop-quark pair production with an additional jet was obtained using CTEQ6M as pdf set, the one for the total top-quark pair cross section with CTEQ6.5.
}

account [36]. It was found that at least this class of higherorder contributions do not change the theoretical prediction dramatically. The main reason is that this type of corrections affect the asymmetric cross section roughly in the same way as the symmetric one. In the ratio the corrections thus cancel to a large extent and lead to a stable theoretical prediction. In $t \bar{t}+$ jet production the asymmetry appears already in the tree amplitude. Thus, the NLO calculation described in this article provides a true NLO prediction for the asymmetry. The calculation presented in this work is an important tool in the experimental analysis of this observable at the Tevatron, where the asymmetry is measured [33, 34]. In a previous letter [37] we reported that the asymmetry receives large corrections. In this paper we study the situation in more detail for various values of the lower cut on the transverse momentum of the hard tagging jet.

As mentioned before, it is expected that the total cross section for top-quark pair production will be measured at the LHC with an accuracy of the order of 5\%. Recently it has been shown in $[26,38,39]$ that the accuracy of the currently available NLO predictions is only at the level of $12 \%$ (at NLO, but further reduced by the inclusion of the threshold logarithms) and largely dominated by the scale uncertainty. In [26] an estimate to the next-to-next-to-leading order (NNLO) cross section has been given. The approximation is based on the assumption that the NNLO corrections will be dominated by the threshold region, as is the case for the NLO corrections. In the threshold region the logarithmic behavior together with the 2-loop Coulomb singularity is derived from general arguments. In addition the complete scale dependence at 2 loops is included in the approximation. Using this approximation to the full NNLO result it is shown in [26] that the theoretical uncertainty may decrease to a few percent. The remaining scale uncertainty is of the same order as the uncertainty induced by the parton distribution functions. Recently some progress towards a complete NNLO calculation has been made [40-48]. The 1-loop corrections to $t \bar{t}+1$-jet constitute an important ingredient to the NNLO calculation of $t \bar{t}$ production at hadron colliders. In this context we mention that-besides our NLO calculation presented here and in [37] — part of the 1-loop amplitudes to $\mathrm{gg} \rightarrow \mathrm{t} \overline{\mathrm{tg}}$ have also been evaluated in [49].

Apart from its significance as a signal process it turns out that $\bar{t}+1$-jet production is also an important background to various new-physics searches. A prominent example is Higgs production via vector-boson fusion. This reaction represents an important discovery channel for a SM Higgs boson with a mass of up to several $100 \mathrm{GeV}[50,51]$. The major background to this reaction is due to $\bar{t}+1$-jet [52], again underlining the need for precise theoretical predictions for this process.

It is well known that predictions at $\mathrm{LO}$ in the coupling constant of QCD are plagued by large uncertainties. In many 
cases the LO predictions in QCD give only a rough estimate. Only by including NLO corrections a quantitatively reliable prediction can be obtained. Given that the conceptual problems in such calculations have been solved since quite some time, one might think that doing the required calculations should be a straightforward task. Unfortunately it turns out that this is not the case. The calculation of radiative corrections for $2 \rightarrow 3$ and $2 \rightarrow 4$ reactions is still non-trivial—not speaking about reactions with an even higher multiplicity. ${ }^{2}$ The complexity of the corresponding matrix elements renders computer codes quite lengthy and CPU time consuming. The (more or less) automatically generated code may in addition lead to numerical instabilities. In particular, the reduction of 1-loop tensor integrals to scalar 1-loop integrals is in general difficult to do in a numerically stable way. In that context the calculation of the 1-loop corrections to top-quark pair production with an additional jet is also interesting as a benchmark process for the development of new methods.

In this paper we extend our previous work [37] on the NLO QCD corrections to $\bar{t}+$ jet production at hadron colliders, where we discussed the scale dependence of the integrated cross sections at the Tevatron and the LHC and of the top-quark charge asymmetry at the Tevatron. We supplement this discussion upon including more numerical results showing the dependence on the lower cut set on the transverse momentum of the hard tagging jet and present first results on differential distributions. Moreover, we provide numerical results on the virtual 1-loop and real-emission corrections for single phase-space points, in order to facilitate future comparisons to our calculation.

This article is organized as follows. In Sect. 2 we briefly describe the calculation of the NLO corrections. Numerical results are presented in Sect. 3. In the appendices we provide numerical results on the virtual and real corrections for individual phase-space points; moreover, we collect the tables with the results for the differential cross sections there.

\section{Outline of the calculation}

\subsection{Born approximation}

In Born approximation the partonic reactions are

$$
\begin{array}{ll}
\mathrm{gg} \rightarrow \mathrm{tt} \mathrm{g}, & q \bar{q} \rightarrow \mathrm{ttg}, \\
q \mathrm{~g} \rightarrow \mathrm{tt} q, & \mathrm{~g} \bar{q} \rightarrow \mathrm{t} \bar{q} .
\end{array}
$$

The last three reactions are related by crossing. Therefore, the required generic matrix elements are

$0 \rightarrow \overline{\operatorname{tg} g g g}, \quad 0 \rightarrow \bar{t} \overline{\mathrm{q}} q \bar{q}$.

\footnotetext{
${ }^{2}$ More details and references on problems and suggested solutions can, for instance, be found in reports like [53, 54].
}

In the following we generically denote the external momenta and helicities by $\left\{p_{i}\right\}$ and $\left\{\lambda_{i}\right\}$ and identify the light partons with $i=1,2,3$. The letter of a specific parton if used as an argument denotes the combination of spin, momentum, and (if relevant) color of this parton, i.e. $\mathrm{t}=\left(p_{\mathrm{t}}, \lambda_{\mathrm{t}}, i_{\mathrm{t}}\right)$ or $\mathrm{g}_{i}=$ $\left(p_{i}, \lambda_{i}, a_{i}\right)$. Representative sets of Born diagrams for the $\mathrm{gg}$ and $q \bar{q}$ channels are depicted in Fig. 2.1. In total, there are $16 \mathrm{LO}$ diagrams for $0 \rightarrow \operatorname{ttggg}$ and 5 for $0 \rightarrow \operatorname{tg} q \bar{q} g$. The color decomposition for a tree amplitude corresponding to the process $0 \rightarrow$ ttggg is

$$
\begin{aligned}
\mathcal{A}_{5}^{(0)}\left(\mathrm{t}, \mathrm{g}_{1}, \mathrm{~g}_{2}, \mathrm{~g}_{3}, \overline{\mathrm{t}}\right)= & g_{\mathrm{s}}^{3} \sum_{\sigma \in S_{3}}\left(T^{a_{\sigma_{1}}} T^{a_{\sigma_{2}}} T^{a_{\sigma_{3}}}\right)_{i_{\mathrm{t}} j_{\mathrm{t}}} \\
& \times A_{5}^{(0)}\left(\mathrm{t}, \mathrm{g}_{\sigma_{1}}, \mathrm{~g}_{\sigma_{2}}, \mathrm{~g}_{\sigma_{3}}, \overline{\mathrm{t}}\right),
\end{aligned}
$$

where $g_{\mathrm{S}}$ is the strong coupling constant, $S_{3}$ the symmetric group and $\sigma=\left(\sigma_{1}, \sigma_{2}, \sigma_{3}\right) \in S_{3}$ a permutation. The generators of the $\mathrm{SU}(\mathrm{N})$ gauge group in the fundamental representation are given by $T^{a}$, and $a_{i}$ is the color index of gluon $\mathrm{g}_{i}$. The sum extends over all permutations in $S_{3}$. Physically this corresponds to all possible color orderings of the gluons. The function $A_{5}^{(0)}\left(\mathrm{t}, \mathrm{g}_{1}, \mathrm{~g}_{2}, \mathrm{~g}_{3}, \overline{\mathrm{t}}\right)$ is thus the colorordered subamplitude, often also called a partial amplitude. Due to the color ordering, only diagrams with a particular ordering contribute to $A_{5}^{(0)}\left(\mathrm{t}, \mathrm{g}_{1}, \mathrm{~g}_{2}, \mathrm{~g}_{3}, \overline{\mathrm{t}}\right)$. The partial amplitudes contain the kinematic information and are individually gauge invariant. The corresponding color decomposition for the process $0 \rightarrow \overline{\mathrm{t}} q \bar{q} \mathrm{~g}$ reads

$$
\begin{aligned}
\mathcal{A}_{5}^{(0)}(\mathrm{t}, \overline{\mathrm{t}}, q, \bar{q}, g)= & g_{\mathrm{s}}^{3}\left[\frac{1}{2} \delta_{i_{\mathrm{t}} j_{\bar{q}}} T_{i_{q} j_{\mathrm{t}}}^{a} A_{5,1}^{(0)}(\mathrm{t}, \overline{\mathrm{t}}, q, \bar{q}, g,)\right. \\
& +\frac{1}{2} \delta_{i_{q} j_{\mathrm{t}}} T_{i_{\mathrm{t}} j_{\bar{q}}}^{a} A_{5,2}^{(0)}(\mathrm{t}, \overline{\mathrm{t}}, q, \bar{q}, g,) \\
& -\frac{1}{2 N} \delta_{i_{\mathrm{t}} j_{\mathrm{t}}} T_{i_{q} j_{\bar{q}}}^{a} A_{5,3}^{(0)}(\mathrm{t}, \overline{\mathrm{t}}, q, \bar{q}, g,) \\
& \left.-\frac{1}{2 N} \delta_{i_{q} j_{\bar{q}}} T_{i_{\mathrm{t}} j_{\mathrm{t}}}^{a} A_{5,4}^{(0)}(\mathrm{t}, \overline{\mathrm{t}}, q, \bar{q}, g,)\right] .
\end{aligned}
$$

We note that the amplitudes $A_{5, i}^{(0)}(i=1,2,3,4)$ are linearly dependent. The relation

$0=A_{5,1}^{(0)}+A_{5,2}^{(0)}-A_{5,3}^{(0)}-A_{5,4}^{(0)}$

can be used to express, for example, $A_{5,4}^{(0)}$ in terms of $A_{5, i}^{(0)}$ with $i=1,2,3$. This is particularly useful for the evaluation of the squared amplitude. Compact analytic results for the LO amplitudes are given in [20], where the amplitudes have been used in the calculation of the NLO corrections for top-quark pair production. In addition we also performed several independent calculations, including one with Madgraph [55], and found complete numerical agreement among all those calculations. 
Fig. 2.1 Representative sets of LO diagrams for $\mathrm{gg}$ fusion and $q \bar{q}$ annihilation in hadronic $\overline{\mathrm{t}}+$ jet production
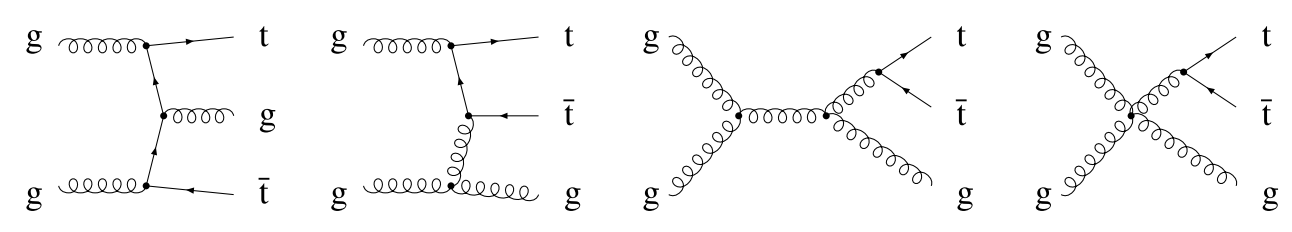
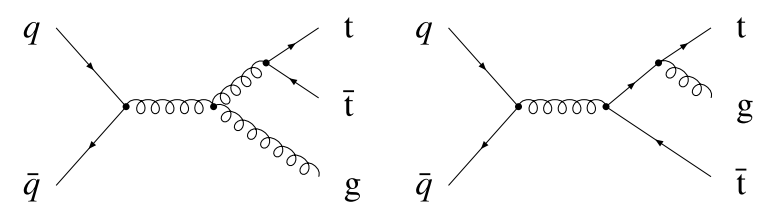

\subsection{Virtual corrections}

The virtual corrections consist of the 1-loop corrections to the LO reactions. One can classify the corrections into selfenergy, vertex, box-type, and pentagon-type corrections, where all the external legs are directly connected to the loop thus forming a pentagon. The latter are the most complicated ones due to their complexity and the involved tensor integrals. Typical examples of the pentagon graphs are shown in Fig. 2.2. Specifically, there are 24 pentagons for $0 \rightarrow \bar{t}$ tggg and 8 for $0 \rightarrow \bar{t} \bar{t} q$ g. The total number of diagrams is 354 for the $0 \rightarrow \overline{\operatorname{tg} g g g}$ case and 94 for the $0 \rightarrow \overline{\mathrm{t}} q \bar{q}$ g case. The challenging step in this context is the numerically fast and stable reduction of the tensor integrals to scalar 1-loop integrals.

Before describing the details we briefly outline the general setup. Owing to the involved kinematics the individual Feynman diagrams lead to large expressions, which are cumbersome to evaluate. To be able to handle the large expressions and to ensure a fast numerical evaluation at the end we used a decomposition of the amplitude according to the spin and color structure. Schematically the decomposition of the 1-loop amplitude $\mathcal{A}_{5}^{(1)}$ reads

$\mathcal{A}_{5}^{(1)}=\sum_{c, s} \mathcal{C}_{c} \times \mathcal{S}_{s}\left(\left\{p_{i}\right\},\left\{\lambda_{i}\right\}\right) \times f_{c s}^{(1)}\left(\left\{p_{i} \cdot p_{j}\right\}\right)$,

where the $\mathcal{C}_{c}$ denote the color structures, $\mathcal{S}_{s}$ are the spin structures (elsewhere called "standard matrix elements"), and the functions $f_{c s}^{(1)}$ are scalar functions that depend only on the scalar products of the external momenta $p_{i}$. In detail, for $0 \rightarrow \bar{t}$ tggg there are 10 independent color structures $\mathcal{C}_{c}$ of the form

$$
\left(T^{a_{\sigma_{1}}} T^{a_{\sigma_{2}}} T^{a_{\sigma_{3}}}\right)_{i_{\mathrm{t}} j_{\mathrm{t}}}, \quad\left(T^{a_{\sigma_{1}}}\right)_{i_{\mathrm{t}} j_{\mathrm{t}}} \delta^{a_{\sigma_{2}} a_{\sigma_{3}},} \quad \mathrm{i} f^{a_{1} a_{2} a_{3}} \delta_{i_{\mathrm{t}} j_{\mathrm{t}}},
$$

where the structure constant of the $\mathrm{SU}(3)$ gauge group $f^{a b c}$ is defined in the usual way through

$\left[T^{a}, T^{b}\right]=\mathrm{i} f^{a b c} T^{c}$.

We note that the counting refers to the group $\mathrm{SU}(3)$; for $\mathrm{SU}(\mathrm{N})$ there are 11 independent structures. For $N=3$ the generic structure $\operatorname{Tr}\left[T^{a_{1}} T^{a_{2}} T^{a_{3}}\right] \delta_{i_{\mathrm{t}} \overline{\mathrm{t}}_{\mathrm{t}}}$ of the $\mathrm{SU}(\mathrm{N})$ case can be further reduced, because $d^{a_{1} a_{2} a_{3}} \delta_{i_{\mathrm{t}} j_{\mathrm{t}}}$, appearing through $\operatorname{Tr}\left[T^{a_{1}} T^{a_{2}} T^{a_{3}}\right]=\frac{1}{4}\left(d^{a_{1} a_{2} a_{3}}+\mathrm{i} f^{a_{1} a_{2} a_{3}}\right)$, is expressible in terms of the other 10 structures within $\mathrm{SU}(3)$ representations. Allowing only for the use of relations which are compatible with conventional dimensional regularization when simplifying the Lorentz structure of the amplitude, we find a few hundred spin structures, such as

$\left[\bar{v}_{\overline{\mathrm{t}}} u_{\mathrm{t}}\right]\left(\varepsilon_{1} \cdot \varepsilon_{2}\right)\left(\varepsilon_{3} \cdot p_{2}\right), \quad\left[\bar{v}_{\overline{\mathrm{t}}} \phi_{3} u_{\mathrm{t}}\right]\left(\varepsilon_{1} \cdot \varepsilon_{2}\right)$,

$\left[\bar{v}_{\bar{t}} \phi_{2} \phi_{3} u_{\mathrm{t}}\right]\left(\varepsilon_{1} \cdot p_{3}\right)$, etc.

where an obvious notation for the Dirac spinors $\bar{v}_{\bar{t}}, u_{\mathrm{t}}$ and gluon-polarization vectors $\varepsilon_{i}$ is used. Restricting ourselves
Fig. 2.2 Representative sets of pentagon diagrams for $\mathrm{gg}$ fusion and $q \bar{q}$ annihilation in hadronic $\overline{\mathrm{t}}+$ jet production at NLO QCD
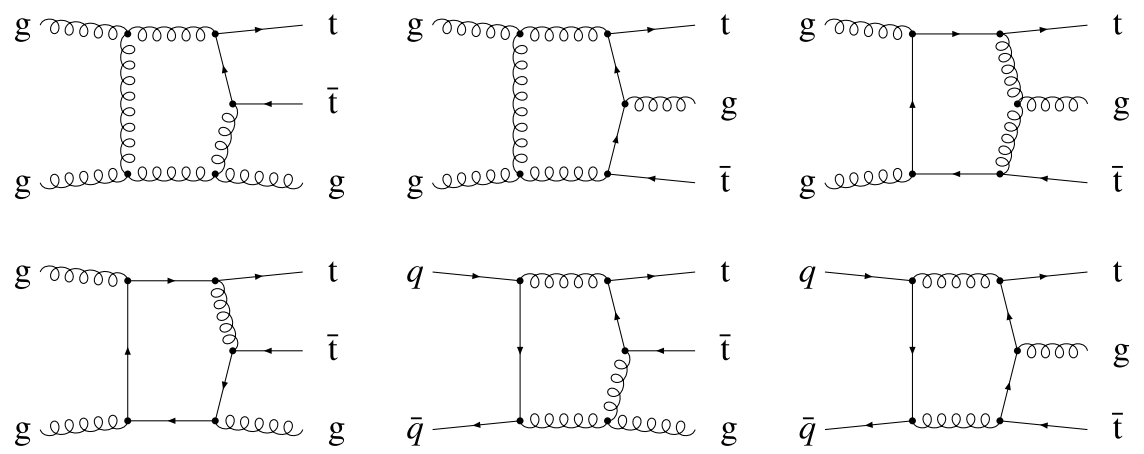
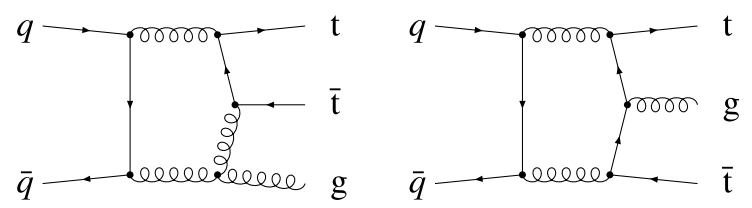
to four dimensions and using explicitly 4-dimensional helicity techniques the number of independent structures may be further reduced. Each gluon-polarization vector can be "gauged" to be orthogonal to an arbitrary light-like reference vector, a fact that reduces the algebraic expressions considerably. A useful choice is, e.g., given by the cyclic set of conditions

$p_{2} \cdot \varepsilon_{1}=p_{3} \cdot \varepsilon_{2}=p_{1} \cdot \varepsilon_{3}=0$,

which supplements the transversality relations $p_{i} \cdot \varepsilon_{i}=0$. However, more than 100 spin structures still remain (in $D$ dimensions) in spite of this simplification. For $0 \rightarrow \overline{\mathrm{t}} q \bar{q} \mathrm{~g}$ there are only 4 color structures (as in LO),

$\delta_{i_{\mathrm{t}} j_{\bar{q}}} T_{i_{q} j_{\overline{\mathrm{t}}}}^{a}, \quad \delta_{i_{q} j_{\overline{\mathrm{t}}}} T_{i_{\mathrm{t}} j_{\bar{q}}}^{a}, \quad \delta_{i_{\mathrm{t}} j_{\mathrm{t}}} T_{i_{q} j_{\bar{q}}}^{a}, \quad \delta_{i_{q} j_{\bar{q}}} T_{i_{\mathrm{t}} j_{\overline{\mathrm{t}}}}^{a}$,

but also more than 100 spin structures, such as

$\left[\bar{v}_{\mathrm{t}} u_{\mathrm{t}}\right]\left[\bar{v}_{\bar{q}} p_{\mathrm{t}} u_{q}\right]\left(\varepsilon \cdot p_{\mathrm{t}}\right), \quad\left[\bar{v}_{\mathrm{t}} u_{\mathrm{t}}\right]\left[\bar{v}_{\bar{q}} \notin u_{q}\right]$,

$\left[\bar{v}_{\mathrm{t}} \gamma^{\mu} u_{\mathrm{t}}\right]\left[\bar{v}_{\bar{q}} \gamma_{\mu} u_{q}\right]\left(\varepsilon \cdot p_{\mathrm{t}}\right)$, etc.

We stress that the algebraic reduction of each Feynman diagram to the standard form shown in (2.5) proceeds in $D=4-2 \epsilon$ space-time dimensions, i.e. only Dirac equations, transversality and gauge conditions of polarization vectors, and momentum conservation are used. We have followed two different strategies for the evaluation of the decomposition shown in (2.5). In one implementation all Feynman diagrams were first combined and then projected onto the different structures. The idea behind this is to form gauge-independent quantities where some cancellations may happen. In the second approach the decomposition is applied to each individual Feynman diagram. In the latter case the numerical evaluation of the functions $f_{c s}$ is most efficiently done if the color structures completely factorizes. For diagrams without 4-gluon vertices this is trivially the case. Diagrams where the number $n_{4}$ of 4-gluon vertices is greater than 0 are decomposed into $3^{n_{4}}$ terms, each with its own color structure. Denoting such 1-loop (sub)graphs generically $\Gamma$, their contributions $f_{c s}^{(\Gamma)}$ to the functions $f_{c s}$ are written as

$f_{c s}^{(\Gamma)}=c_{c}^{(\Gamma)} f_{s}^{(\Gamma)}$

with constants $c_{c}^{(\Gamma)}$, i.e. the total color structure of $\Gamma$ is $\sum_{c} \mathcal{C}_{c} c_{c}^{(\Gamma)}$. The color-stripped functions $f_{s}^{(\Gamma)}$, which contain the time-consuming loop functions are the same for all color channels. Writing the LO amplitude as

$\mathcal{A}_{5}^{(0)}=\sum_{c} \mathcal{C}_{c} A_{5, c}^{(0)}\left(\left\{p_{i}\right\},\left\{\lambda_{i}\right\}\right)$, the contribution of $\Gamma$ to the 1-loop-corrected spin- and color-averaged squared amplitude is evaluated as follows:

$$
\begin{aligned}
& \sum_{\text {color, spin }} 2 \operatorname{Re}\left\{\mathcal{A}_{5}^{(0) *} \mathcal{A}_{5}^{(\Gamma)}\right\} \\
& =2 \operatorname{Re}\left\{\sum_{c, c^{\prime}} C_{c c^{\prime}} c_{c^{\prime}}^{(\Gamma)} \sum_{s} f_{s}^{(\Gamma)}\left(\left\{p_{i} \cdot p_{j}\right\}\right)\right. \\
& \left.\quad \times \sum_{\left\{\lambda_{i}\right\}} A_{5, c}^{(0)}\left(\left\{p_{i}\right\},\left\{\lambda_{i}\right\}\right)^{*} \mathcal{S}_{s}\left(\left\{p_{i}\right\},\left\{\lambda_{i}\right\}\right)\right\} .
\end{aligned}
$$

In detail, the color correlation matrix

$C_{c c^{\prime}}=\sum_{\text {color }} \mathcal{C}_{c}^{\dagger} \mathcal{C}_{c^{\prime}}$

is calculated only once and for all for the whole process, and the interferences $A_{5, c}^{(0)}{ }^{*} \mathcal{S}_{s}$ of the LO amplitude and the different spin structures $\mathcal{S}_{S}$ are only calculated once per phasespace point for every spin state. The approach in which first all the diagrams are combined and then the projection is done yields similar formulae. The final formula then reads

$$
\begin{aligned}
& \sum_{\text {color, spin }} 2 \operatorname{Re}\left\{\left(\mathcal{A}_{5}^{(0)}\right)^{\dagger} \mathcal{A}_{5}\right\} \\
& =2 \operatorname{Re}\left\{\sum_{\left\{\lambda_{i}\right\}} \sum_{c, c^{\prime}} \sum_{s, s^{\prime}} C_{c c^{\prime}} f_{c s}\left(\left\{p_{i} \cdot p_{j}\right\}\right) f_{c^{\prime} s^{\prime}}^{(0) *}\left(\left\{p_{i} \cdot p_{j}\right\}\right)\right. \\
& \left.\quad \times \mathcal{S}_{s}\left(\left\{p_{i}\right\},\left\{\lambda_{i}\right\}\right) \mathcal{S}_{s^{\prime}}^{*}\left(\left\{p_{i}\right\},\left\{\lambda_{i}\right\}\right)\right\}
\end{aligned}
$$

where $f_{c s}^{(0)}$ are the scalar functions appearing in the decomposition of the Born amplitude. To ensure the correctness of our results the two slightly different approaches were implemented in two complete independent computer codes. We note at this point that no significant difference between the two approaches concerning speed and numerical stability was observed. We also note that we used as far as possible different methods and also different tools to obtain the various ingredients discussed above. In the following we give some details of the techniques employed in the two implementations.

Version 1 of the virtual corrections is essentially obtained following the method described in [23], where $\mathrm{tt}^{\mathrm{H}} \mathrm{H}$ production at hadron colliders was considered. Feynman diagrams and amplitudes have been generated with the Fey$n$ Arts package $[56,57]$ and further processed with in-house Mathematica routines, which automatically create an output in Fortran. The infrared (IR), i.e. soft and collinear, singularities - which are treated in dimensional regularization in both calculations - are analytically separated from the finite remainder in terms of triangle subdiagrams, as described in $[23,58]$. This separation, in particular, allows for 
a transparent evaluation of so-called rational terms, which originate from $D$-dependent terms multiplying IR divergences, which appear as single or double poles in $(D-4)$. As generally shown in [59], after properly separating IR from ultraviolet (UV) divergences such rational terms originating from IR divergences completely cancel; this general result is confirmed in our explicit calculation. The tensor integrals appearing in the pentagon diagrams are directly reduced to box integrals following [60]. (Similar methods have been proposed in [61].) This method does not introduce inverse Gram determinants in this step, thereby avoiding notorious numerical instabilities in regions where these determinants become small. Box and lower-point integrals are reduced à la Passarino-Veltman [62] to scalar integrals, which are either calculated analytically or using the results of [63-65]. Sufficient numerical stability is already achieved in this way. Nevertheless the integral evaluation is currently further refined by employing the more sophisticated methods described in [66] in order to numerically stabilize the tensor integrals in exceptional phase-space regions.

Version 2 of the evaluation of loop diagrams starts with the generation of diagrams and amplitudes via $Q G R A F$ [67], which are then further manipulated with Form [68] and Maple and eventually automatically translated into $\mathrm{C}++$ code. The reduction of the 5-point tensor integrals to scalar integrals is performed with an extension of the method described in [69]. In this procedure also inverse Gram determinants of 4 4-momenta are avoided. The lower-point tensor integrals are reduced using an independent implementation of the Passarino-Veltman procedure. The IR-finite scalar integrals are evaluated using the $F F$ package [70, 71]. Although the entire procedure is sufficiently stable, further numerical stabilization of the tensor reduction is planned following the expansion techniques suggested in [72] for exceptional phase-space regions.

As stated above, we used dimensional regularization to regularize UV as well as soft and collinear divergences. We renormalized the coupling in a mixed scheme in which the light flavors are treated according to the modified minimal subtraction $\overline{\mathrm{MS}}$, while the top-quark loop of the gluon selfenergy is subtracted at zero momentum. The top-quark mass is renormalized in the on-shell scheme. More specifically we used the renormalization constants as given for example in [23]. In these formulae the divergences of UV and IR origin are separated. They allow us to check UV and IR finiteness separately.

\subsection{Real corrections}

The generic matrix elements for the real corrections are given by

$0 \rightarrow \mathrm{t} \overline{\mathrm{t}} g g g g, \quad 0 \rightarrow \mathrm{t} \overline{\mathrm{t}} q \bar{q} g g$,
$0 \rightarrow \mathrm{t} \overline{\mathrm{t}} q \bar{q} q^{\prime} \bar{q}^{\prime}, \quad 0 \rightarrow \overline{\mathrm{t}} q \bar{q} q \bar{q}$, with $q \neq q^{\prime}$. The various partonic processes are obtained from these matrix elements by all possible crossings of light particles into the initial state. While the crossing symmetry is extremely helpful in constructing the required amplitudes, it should be kept in mind that the large number of possible channels obtained from the different crossings lead to a significant increase in the computational complexity, given that every channel has to be integrated over the phase space. The amplitude for the process $0 \rightarrow \operatorname{tt} q \bar{q} q \bar{q}$ with identical quarks $q$ can be obtained from the amplitude of the process $0 \rightarrow \operatorname{t} \bar{q} q q^{\prime} \bar{q}^{\prime}$ with non-identical quarks $q$ and $q^{\prime}$ :

$$
\begin{aligned}
\mathcal{A}_{6}^{(0)}(\mathrm{t}, \overline{\mathrm{t}}, q, \bar{q}, q, \bar{q})= & \mathcal{A}_{6}^{(0)}\left(\mathrm{t}, \overline{\mathrm{t}}, q, \bar{q}, q^{\prime}, \bar{q}^{\prime}\right) \\
& -\mathcal{A}_{6}^{(0)}\left(\mathrm{t}, \overline{\mathrm{t}}, q, \bar{q}^{\prime}, q^{\prime}, \bar{q}\right) .
\end{aligned}
$$

The color decomposition of a tree amplitude corresponding to the process $0 \rightarrow \bar{t}$ tgggg is

$$
\begin{aligned}
\mathcal{A}_{6}^{(0)}\left(\mathrm{t}, \mathrm{g}_{1}, \mathrm{~g}_{2}, \mathrm{~g}_{3}, \mathrm{~g}_{4}, \overline{\mathrm{t}}\right)= & g_{\mathrm{s}}^{4} \sum_{\sigma \in S_{4}}\left(T^{a_{\sigma_{1}}} T^{a_{\sigma_{2}}} T^{a_{\sigma_{3}}} T^{a_{\sigma_{4}}}\right)_{i_{\mathrm{t}} j_{\mathrm{t}}} \\
& \times A_{6}^{(0)}\left(\mathrm{t}, \mathrm{g}_{\sigma_{1}}, \mathrm{~g}_{\sigma_{2}}, \mathrm{~g}_{\sigma_{3}}, \mathrm{~g}_{\sigma_{4}}, \overline{\mathrm{t}}\right),
\end{aligned}
$$

where the sum is over all permutations $\sigma=\left(\sigma_{1}, \sigma_{2}, \sigma_{3}, \sigma_{4}\right)$ of the symmetric group $S_{4}$. The color decomposition for the process $0 \rightarrow \operatorname{tt} q \bar{q} g \mathrm{~g}$ reads

$$
\begin{aligned}
\mathcal{A}_{6}^{(0)}\left(\mathrm{t}, \overline{\mathrm{t}}, q, \bar{q}, \mathrm{~g}_{1}, \mathrm{~g}_{2}\right) & \\
= & \frac{g_{\mathrm{s}}^{4}}{2} \sum_{\left(\sigma_{1} \sigma_{2}\right) \in S_{2}}\left[\delta_{i_{\mathrm{t}} j_{\bar{q}}}\left(T^{a_{\sigma_{1}}} T^{a_{\sigma_{2}}}\right)_{i_{q} j_{\overline{\mathrm{t}}}} A_{6,1}^{(0)}\left(\mathrm{t}, \overline{\mathrm{t}}, q, \bar{q}, \mathrm{~g}_{\sigma_{1}}, \mathrm{~g}_{\sigma_{2}}\right)\right. \\
& +T_{i_{\mathrm{t}} \bar{j}_{\bar{q}}}^{a_{\sigma_{1}}} T_{i_{q} \bar{j}_{\overline{\mathrm{t}}}}^{a_{\sigma_{2}}} A_{6,2}^{(0)}\left(\mathrm{t}, \overline{\mathrm{t}}, q, \bar{q}, \mathrm{~g}_{\sigma_{1}}, \mathrm{~g}_{\sigma_{2}}\right) \\
& +\left(T^{a_{\sigma_{1}}} T^{a_{\sigma_{2}}}\right)_{i_{\mathrm{t}} j_{\bar{q}}} \delta_{i_{q} j_{\mathrm{t}}} A_{6,3}^{(0)}\left(\mathrm{t}, \overline{\mathrm{t}}, q, \bar{q}, \mathrm{~g}_{\sigma_{1}}, \mathrm{~g}_{\sigma_{2}}\right) \\
& -\frac{1}{N} \delta_{i_{\mathrm{t}} j_{\mathrm{t}}}\left(T^{a_{\sigma_{1}}} T^{a_{\sigma_{2}}}\right)_{i_{q} j_{\bar{q}}} A_{6,4}^{(0)}\left(\mathrm{t}, \overline{\mathrm{t}}, q, \bar{q}, \mathrm{~g}_{\sigma_{1}}, \mathrm{~g}_{\sigma_{2}}\right) \\
& -\frac{1}{N} T_{i_{\mathrm{t}} j_{\overline{\mathrm{t}}}}^{a_{\sigma_{1}}} T_{i_{q} j_{\bar{q}}}^{a_{\sigma_{2}}} A_{6,5}^{(0)}\left(\mathrm{t}, \overline{\mathrm{t}}, q, \bar{q}, \mathrm{~g}_{\sigma_{1}}, \mathrm{~g}_{\sigma_{2}}\right) \\
& \left.-\frac{1}{N}\left(T^{a_{\sigma_{1}}} T^{a_{\sigma_{2}}}\right)_{i_{\mathrm{t}} j_{\overline{\mathrm{t}}}} \delta_{i_{q} j_{\bar{q}}} A_{6,6}^{(0)}\left(\mathrm{t}, \overline{\mathrm{t}}, q, \bar{q}, \mathrm{~g}_{\sigma_{1}}, \mathrm{~g}_{\sigma_{2}}\right)\right]
\end{aligned}
$$

where again the sum is over all permutations of the gluon legs. Finally, the color decomposition of the process $0 \rightarrow$ $\overline{\mathrm{t}} q \bar{q} q^{\prime} \bar{q}^{\prime}$ is

$$
\begin{aligned}
& \mathcal{A}_{6}^{(0)}\left(\mathrm{t}, \overline{\mathrm{t}}, q, \bar{q}, q^{\prime}, \bar{q}^{\prime}\right) \\
& \quad=\frac{g_{\mathrm{s}}^{4}}{4}\left[\delta_{i_{\mathrm{t}} j_{\bar{q}}} \delta_{i_{q} j_{\bar{q}^{\prime}}} \delta_{i_{q^{\prime}} j_{\mathrm{t}}} A_{6,1}^{(0)}\left(\mathrm{t}, \overline{\mathrm{t}}, q, \bar{q}, q^{\prime}, \bar{q}^{\prime}\right)\right.
\end{aligned}
$$




$$
\begin{aligned}
& +\delta_{i_{\mathrm{t}} j_{\bar{q}^{\prime}}} \delta_{i_{q^{\prime}} j_{\bar{q}}} \delta_{i_{q} j_{\mathrm{t}}} A_{6,1}^{(0)}\left(\mathrm{t}, \overline{\mathrm{t}}, q^{\prime}, \bar{q}^{\prime}, q, \bar{q}\right) \\
& -\frac{1}{N} \delta_{i_{\mathrm{t}} j_{\bar{q}}} \delta_{i_{q} j_{\overline{\mathrm{t}}}} \delta_{i_{q^{\prime}} j_{\bar{q}^{\prime}}} A_{6,2}^{(0)}\left(\mathrm{t}, \overline{\mathrm{t}}, q, \bar{q}, q^{\prime}, \bar{q}^{\prime}\right) \\
& -\frac{1}{N} \delta_{i_{\mathrm{t}} j_{\bar{q}^{\prime}}} \delta_{i_{q^{\prime}} \bar{j}_{\mathrm{t}}} \delta_{i_{q} j_{\bar{q}}} A_{6,2}^{(0)}\left(\mathrm{t}, \overline{\mathrm{t}}, q^{\prime}, \bar{q}^{\prime}, q, \bar{q}\right) \\
& -\frac{1}{N} \delta_{i_{q} j_{\bar{q}^{\prime}}} \delta_{i_{q^{\prime}} j_{q}} \delta_{i_{\mathrm{t}} j_{\mathrm{t}}} A_{6,3}^{(0)}\left(\mathrm{t}, \overline{\mathrm{t}}, q, \bar{q}, q^{\prime}, \bar{q}^{\prime}\right) \\
& \left.+\frac{1}{N^{2}} \delta_{i_{\mathrm{t}} j_{\overline{\mathrm{t}}}} \delta_{i_{q} j_{\bar{q}}} \delta_{q_{q^{\prime}} \bar{q}_{\bar{q}^{\prime}}} A_{6,4}^{(0)}\left(\mathrm{t}, \overline{\mathrm{t}}, q, \bar{q}, q^{\prime}, \bar{q}^{\prime}\right)\right] .
\end{aligned}
$$

To extract the IR singularities and for their combination with the virtual corrections we employ the dipole subtraction formalism [73-75]. Specifically, the formulation [75] for massive quarks is used. At NLO schematically one has the following contributions:

$$
\langle O\rangle^{\mathrm{NLO}}=\int_{n+1} O_{n+1} \mathrm{~d} \sigma^{\mathrm{R}}+\int_{n} O_{n} \mathrm{~d} \sigma^{\mathrm{V}}+\int_{n} O_{n} \mathrm{~d} \sigma^{\mathrm{C}} .
$$

Here $\mathrm{d} \sigma^{\mathrm{R}}$ denotes the real-emission contribution, whose matrix elements are given by the square of the Born amplitudes with 6 partons $\left|\mathcal{A}_{6}^{(0)}\right|^{2}, \mathrm{~d} \sigma^{\mathrm{V}}$ is the virtual contribution, whose matrix elements are given by the interference term of the 1-loop amplitudes $\mathcal{A}_{5}^{(1)}$ with 5 partons with the corresponding Born amplitude $\mathcal{A}_{5}^{(0)}$, and $\mathrm{d} \sigma^{\mathrm{C}}$ denotes a collinear subtraction term, which originates from the factorization of the initial-state collinear singularities. The function $O_{n}$ defined on the $n$-particle phase space stands for any prescription $(\theta$-functions for phase-space cuts, $\delta$-functions for distributions) defining an IR-safe observable. Taken separately, the individual contributions are IR divergent, and only their sum is finite. In order to render the individual contributions finite, so that the phase-space integrations can be performed by Monte Carlo methods, one adds and subtracts a suitably chosen "counterterm" $\mathrm{d} \sigma$ A.

$$
\begin{aligned}
\langle O\rangle^{\mathrm{NLO}}= & \int_{n+1}\left(O_{n+1} \mathrm{~d} \sigma^{\mathrm{R}}-O_{n} \mathrm{~d} \sigma^{\mathrm{A}}\right) \\
& +\int_{n}\left(O_{n} \mathrm{~d} \sigma^{\mathrm{V}}+O_{n} \mathrm{~d} \sigma^{\mathrm{C}}+O_{n} \int_{1} \mathrm{~d} \sigma^{\mathrm{A}}\right) .
\end{aligned}
$$

The matrix element corresponding to the approximation term $\mathrm{d} \sigma^{\mathrm{A}}$ is given as a sum over dipoles:

$$
\mathrm{d} \sigma^{\mathrm{A}} \propto \sum_{\text {pairs } i, j} \sum_{k \neq i, j} \mathcal{D}_{i j, k}
$$

Each dipole contribution has the following form:

$$
\begin{aligned}
\mathcal{D}_{i j, k}= & -\frac{1}{2 p_{i} \cdot p_{j}} \mathcal{A}_{5}^{(0) *}\left(p_{1}, \ldots, \tilde{p}_{(i j)}, \ldots, \tilde{p}_{k}, \ldots\right) \\
& \times \frac{\mathbf{T}_{k} \cdot \mathbf{T}_{i j}}{\mathbf{T}_{i j}^{2}} V_{i j, k} \mathcal{A}_{5}^{(0)}\left(p_{1}, \ldots, \tilde{p}_{(i j)}, \ldots, \tilde{p}_{k}, \ldots\right) .
\end{aligned}
$$

Here $\mathbf{T}_{i}$ denotes the color charge operator for parton $i$ and $V_{i j, k}$ is a matrix in the spin space of the emitter parton $(i j)$. The momenta $\tilde{p}_{(i j)}$ and $\tilde{p}_{k}$ are obtained from the momenta $p_{i}, p_{j}$ and $p_{k}$. In general, the operators $\mathbf{T}_{i}$ lead to color correlations, while the $V_{i j, k}$ may lead to spin correlations. The approximation $\mathrm{d} \sigma^{\mathrm{A}}$ has to fulfill the requirement that $\mathrm{d} \sigma^{\mathrm{A}}$ is a proper approximation of $\mathrm{d} \sigma^{\mathrm{R}}$ with the same point-wise singular behavior (in $D=4-2 \epsilon$ dimensions) as $\mathrm{d} \sigma^{\mathrm{R}}$ itself. Thus, $\mathrm{d} \sigma^{\mathrm{A}}$ acts as a local counterterm for $\mathrm{d} \sigma^{\mathrm{R}}$, and one can safely perform the limit $\epsilon \rightarrow 0$. This defines the finite contribution

$$
\langle O\rangle_{\{n+1\}}^{\mathrm{NLO}}=\int_{n+1}\left(\left.O_{n+1} \mathrm{~d} \sigma^{\mathrm{R}}\right|_{\epsilon=0}-\left.O_{n} \mathrm{~d} \sigma^{\mathrm{A}}\right|_{\epsilon=0}\right) .
$$

The subtraction term can be integrated over the unresolved 1-parton phase space. Due to this integration, all spin correlations average out, but color correlations still remain. In a compact notation, the result of this integration is often written as

$\mathrm{d} \sigma^{\mathrm{C}}+\int_{1} \mathrm{~d} \sigma^{\mathrm{A}}=\mathbf{I} \otimes \mathrm{d} \sigma^{\mathrm{B}}+\mathbf{K} \otimes \mathrm{d} \sigma^{\mathrm{B}}+\mathbf{P} \otimes \mathrm{d} \sigma^{\mathrm{B}}$.

The notation $\otimes$ indicates that color correlations still remain and that an integration is involved. The term $\mathbf{I} \otimes \mathrm{d} \sigma^{\mathrm{B}}$ lives on the phase space of the Born configuration and has the appropriate singularity structure to cancel the IR divergences coming from the 1-loop amplitude. Therefore, $\mathrm{d} \sigma^{\mathrm{V}}+\mathbf{I} \otimes \mathrm{d} \sigma^{\mathrm{B}}$ is IR finite. The terms $(\mathbf{K}+\mathbf{P}) \otimes \mathrm{d} \sigma^{\mathrm{B}}$ involve in addition an integration over the momentum fraction $x$ that rules the collinear splitting of the incoming parton. From the integration of the subtraction terms we obtain the finite contribution

$$
\begin{aligned}
\langle O\rangle_{\{n\}}^{\mathrm{NLO}}= & \int_{n} O_{n}\left(\mathrm{~d} \sigma^{\mathrm{V}}+\mathbf{I} \otimes \mathrm{d} \sigma^{\mathrm{B}}\right. \\
& \left.+\mathbf{K} \otimes \mathrm{d} \sigma^{\mathrm{B}}+\mathbf{P} \otimes \mathrm{d} \sigma^{\mathrm{B}}\right)_{\varepsilon=0} .
\end{aligned}
$$

The final structure of an NLO calculation in the subtraction formalism is then

$$
\langle O\rangle^{\mathrm{NLO}}=\langle O\rangle_{\{n+1\}}^{\mathrm{NLO}}+\langle O\rangle_{\{n\}}^{\mathrm{NLO}} .
$$

Since both contributions on the right-hand side of (2.25) are now finite, they can be evaluated with numerical methods. The explicit forms of the dipole terms $\mathcal{D}_{i j, k}$, together with the integrated counterparts, can be found in [73] for massless QCD and in [74, 75] including massive quarks. 
Analogously to our evaluation of the virtual corrections, we have also performed two independent calculations of the real corrections.

One calculation of the real corrections results from a fully automated calculation based on helicity amplitudes, as described in [76]. Individual helicity amplitudes are computed with the help of Berends-Giele recurrence relations [77]. The evaluation of color factors and the generation of subtraction terms is automated. For the channel $\mathrm{gg} \rightarrow \overline{\mathrm{t} g g}$ a dedicated soft-insertion routine [78] is used for the generation of the phase space.

The second calculation uses for the LO $2 \rightarrow 3$ processes and the $\mathrm{gg} \rightarrow \overline{\mathrm{t}} \mathrm{gg}$ process optimized code obtained from a Feynman diagrammatic approach. As in the calculation described before, standard techniques like color decomposition and the use of helicity amplitudes are employed. For the $2 \rightarrow 4$ processes including light quarks, Madgraph [55] has been used. The subtraction terms according to [75] are obtained in a semi-automatized manner based on a library written in $\mathrm{C}++$.

The two independent computer codes were compared point-wise at a few phase-space points. In addition, the entire numerical integration of the real corrections was done independently using the two codes. We found complete agreement of the numerical results when the numerical uncertainty from the phase-space integration is taken into account.

\section{Numerical results}

\subsection{Setup}

In the following we consistently use the CTEQ6 [79] set of parton distribution functions (PDFs). In detail, we take CTEQ6L1 PDFs with a 1-loop running $\alpha_{\mathrm{s}}$ in LO and CTEQ6M PDFs with a 2-loop running $\alpha_{\mathrm{s}}$ in NLO. The number of active flavors is $N_{\mathrm{F}}=5$, and the respective QCD parameters are $\Lambda_{5}^{\mathrm{LO}}=165 \mathrm{MeV}$ and $\Lambda_{5}^{\overline{\mathrm{MS}}}=226 \mathrm{MeV}$. As mentioned earlier, the top-quark loop in the gluon self-energy is subtracted at zero momentum. In this scheme the running of $\alpha_{\mathrm{s}}$ is generated solely by the contributions of the light quark and gluon loops. The top-quark mass is renormalized in the on-shell scheme; as numerical value we take $m_{\mathrm{t}}=174 \mathrm{GeV}$.
If not stated otherwise, we identify the renormalization and factorization scales, $\mu_{\text {ren }}$ and $\mu_{\text {fact }}$, with $m_{\mathrm{t}}$.

For the definition of the tagged hard jet we apply the jet algorithm of [80] with $R=1$ and require a transverse mo-

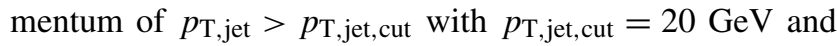
$p_{\mathrm{T}, \mathrm{jet} \text {,cut }}=50 \mathrm{GeV}$ for the hardest jet at the Tevatron and the LHC, respectively. The outgoing (anti)top quarks are neither affected by the jet algorithm nor by the phase-space cut. We assume them to be always tagged. Note that the LO prediction and the virtual corrections are not influenced by the recombination procedure of the jet algorithm, but the real corrections are.

Up to the transverse-momentum cut $p_{\mathrm{T} \text {, jet,cut }}=50 \mathrm{GeV}$ for the LHC, the setup used in this article coincides with the one used in [37]. There, $p_{\mathrm{T}, \text { jet,cut }}=20 \mathrm{GeV}$ was used both for the Tevatron and the LHC.

\subsection{Results for the Tevatron}

As discussed in [37], the integrated LO cross section for $\overline{\mathrm{t}}+1$-jet production at the Tevatron is dominated by the $q \bar{q}$ channel with about $85 \%$, followed by the gg channel with about $7 \%$. This is rather similar to the inclusive top-quark pair cross section, where again at LO about $90 \%$ is obtained from the $q \bar{q}$ channel and about $10 \%$ from the gg channel. Different from the inclusive case, the $q \mathrm{~g}(\bar{q} \mathrm{~g})$ are not suppressed in the coupling. This accounts for the slightly larger contribution from these channels. One should keep in mind that the precise contribution of individual channels depends on the factorization scale as well as on the chosen parton distributions. As a consequence the aforementioned numbers give just a qualitative picture.

In Table 3.1 we provide the LO and NLO predictions for the integrated cross sections for different values of the cut on the transverse momentum of the hard jet (left part). The values presented are for the central scale $\mu=\mu_{\text {fact }}=\mu_{\text {ren }}=$ $m_{\mathrm{t}}$. In parentheses we quote the uncertainty due to the numerical integration. The scale dependence is indicated by the upper and lower indices. The upper (lower) index represents the change when the scale is shifted towards $\mu=m_{\mathrm{t}} / 2$ $\left(\mu=2 m_{\mathrm{t}}\right)$. Rescaling the common scale $\mu=\mu_{\text {fact }}=\mu_{\text {ren }}$ from the default value $m_{\mathrm{t}}$ up (down) by a factor 2 changes the cross section in LO and NLO by about $60 \%$ (35\%) and
Table 3.1 Cross section $\sigma_{\text {tijet }}$ and forward-backward charge asymmetry $A_{\mathrm{FB}}^{\mathrm{t}}$ at the Tevatron for different values of $p_{\mathrm{T}, \text { jet,cut }}$ for $\mu=\mu_{\text {fact }}=\mu_{\text {ren }}=m_{\mathrm{t}}$. The upper and lower indices are the shifts towards $\mu=m_{\mathrm{t}} / 2$ and $\mu=2 m_{\mathrm{t}}$

\begin{tabular}{|c|c|c|c|c|}
\hline \multirow[t]{2}{*}{$p_{\mathrm{T}, \mathrm{jet}, \mathrm{cut}}[\mathrm{GeV}]$} & \multicolumn{2}{|l|}{$\sigma_{\mathrm{ttjjet}}[\mathrm{pb}]$} & \multicolumn{2}{|l|}{$A_{\mathrm{FB}}^{\mathrm{t}}[\%]$} \\
\hline & LO & NLO & LO & NLO \\
\hline 20 & $1.583(2)_{-0.55}^{+0.96}$ & $1.791(1)_{-0.31}^{+0.16}$ & $-7.69(4)_{-0.085}^{+0.10}$ & $-1.77(5)_{-0.30}^{+0.58}$ \\
\hline 30 & $0.984(1)_{-0.34}^{+0.60}$ & $1.1194(8)_{-0.20}^{+0.11}$ & $-8.29(5)_{-0.085}^{+0.12}$ & $-2.27(4)_{-0.51}^{+0.31}$ \\
\hline 40 & $0.6632(8)_{-0.23}^{+0.41}$ & $0.7504(5)_{-0.14}^{+0.072}$ & $-8.72(5)_{-0.10}^{+0.13}$ & $-2.73(4)_{-0.49}^{+0.35}$ \\
\hline 50 & $0.4670(6)_{-0.17}^{+0.29}$ & $0.5244(4)_{-0.096}^{+0.049}$ & $-8.96(5)_{-0.11}^{+0.14}$ & $-3.05(4)_{-0.39}^{+0.49}$ \\
\hline
\end{tabular}


$9 \%(18 \%)$, respectively, i.e. the scale uncertainty is reduced considerably through the inclusion of the NLO corrections. The above findings are rather insensitive to the chosen cut value. We find only variations at the percent level. In particular, there is no big difference for the lowest cut value compared to the other values, suggesting that the perturbative expansion is under control and not spoiled by the appearance of large logarithms. Compared with the total cross section, we find that for the small $p_{\mathrm{T}}$ cut of $20 \mathrm{GeV}$ the $\overline{\mathrm{t}}+1$-jet events represent almost $30 \%$ of the total cross section. This fraction shows an evident dependence on the value chosen for the $p_{\mathrm{T}}$ cut. The fraction is reduced to about $8 \%$ when $50 \mathrm{GeV}$ is chosen for the cut. The NLO corrections change the ratio for a given value of $p_{\mathrm{T}}$ cut only at the level of a few percent.

In the right part of Table 3.1 we show results for the forward-backward charge asymmetry. In LO the top-quark charge asymmetry is defined by

$A_{\mathrm{FB}, \mathrm{LO}}^{\mathrm{t}}=\frac{\sigma_{\mathrm{LO}}^{-}}{\sigma_{\mathrm{LO}}^{+}}$,

with the definition

$\sigma_{\mathrm{LO}}^{ \pm}=\sigma_{\mathrm{LO}}\left(y_{\mathrm{t}}>0\right) \pm \sigma_{\mathrm{LO}}\left(y_{\mathrm{t}}<0\right)$,

where $y_{\mathrm{t}}$ denotes the rapidity of the top quark. Cross-section contributions $\sigma\left(y_{\mathrm{t}} \geq 0\right)$ correspond to top quarks in the forward or backward hemispheres, respectively, where incoming protons fly into the forward direction by definition. Denoting the corresponding NLO contributions to the cross sections by $\delta \sigma_{\mathrm{NLO}}^{ \pm}$, we define the asymmetry at NLO by

$A_{\mathrm{FB}, \mathrm{NLO}}^{\mathrm{t}}=\frac{\sigma_{\mathrm{LO}}^{-}}{\sigma_{\mathrm{LO}}^{+}}\left(1+\frac{\delta \sigma_{\mathrm{NLO}}^{-}}{\sigma_{\mathrm{LO}}^{-}}-\frac{\delta \sigma_{\mathrm{NLO}}^{+}}{\sigma_{\mathrm{LO}}^{+}}\right)$,

i.e. via a consistent expansion in $\alpha_{\mathrm{s}}$. Note, however, that the LO cross sections in (3.3) are evaluated in the NLO setup $\left(\mathrm{PDFs}, \alpha_{\mathrm{s}}\right)$. In [37] it was already pointed out that the LO asymmetry for a $p_{\mathrm{T}}$-cut of $20 \mathrm{GeV}$, which is about $-7.7 \%$ with a small scale uncertainty, is reduced to about $-1.8 \%$ with the rather large scale uncertainty that is-assessed conservatively-almost as large as its absolute size. The reason for this growing scale uncertainty when going from LO to NLO simply results from the fact that the LO prediction for $A_{\mathrm{FB}}^{\mathrm{t}}$ is independent of the renormalization scale, since the strong coupling drops out in the ratio. Thus, the scale dependence does not reflect the total theoretical uncertainty in LO at all for this quantity. Table 3.1 shows that this feature qualitatively holds true also for larger values of $p_{\mathrm{T} \text {,jet,cut }}$ used for the jet definition, but the LO and NLO asymmetries are shifted towards larger absolute values for a larger cut.

Figure 3.1 shows the distributions in the transverse momenta of the hard jet, $p_{\mathrm{T}, \text { jet }}$, of the total $\overline{\mathrm{t}}$ system, $p_{\mathrm{T}, \mathrm{t}}$, and of the top quark, $p_{\mathrm{T}, \mathrm{t}}$. In LO $p_{\mathrm{T}, \mathrm{jet}}$ and $p_{\mathrm{T}, \mathrm{tt}}$ coincide because of momentum conservation in the transverse plane, but the radiation of 2 jets in the real corrections renders them different. The numerical results in Fig. 3.1, however, reveal that the differences are very small. The shown $p_{\mathrm{T}}$ distributions drop with growing $p_{\mathrm{T}}$, where the spectrum for the top quark is much harder than the ones for the jet and the t⿱t tem. In the $p_{\mathrm{T}, \mathrm{jet}}$ and $p_{\mathrm{T}, \mathrm{tt}}$ spectra $93 \%$ of the events are concentrated below a $p_{\mathrm{T}}$ of about $100 \mathrm{GeV}$ at NLO, while $92 \%$ of the events have a $p_{\mathrm{T}, \mathrm{t}}$ with less than $200 \mathrm{GeV}$. Employing a fixed scale $\mu=m_{\mathrm{t}}$, the NLO corrections do not simply rescale the LO shape but induce distortions at the level of some $10 \%$, which redistribute events from larger to smaller transverse momenta. We believe that two effects contribute to these distortions. First of all, the use of a fixed renormalization scale $\mu_{\text {ren }}=m_{\mathrm{t}}$ is not an appropriate choice for high $p_{\mathrm{T}}$ events. Due to the large value of $\alpha_{\mathrm{s}}$ the LO calculation overestimates the cross section for high $p_{\mathrm{T}}$ and the NLO calculation has to compensate this scale choice. We expect that the distortions due to this effect are reduced if an appropriate $p_{\mathrm{T}}$-dependent scale choice is used, such as $\mu=\sqrt{p_{\mathrm{T}}^{2}+m_{\mathrm{t}}^{2}}$. As a second effect in particular the $p_{\mathrm{T}}$ distribution of the top quarks can become softer due to the emission of additional particles, which is accounted for the first time by an NLO calculation.

As can also be seen from the lower panel of each plot, we find again an important reduction of the scale dependence when the NLO corrections are taken into account. At least for the $p_{\mathrm{T}, \mathrm{jet}}{ }^{-}$and $p_{\mathrm{T}, \mathrm{tt}}$ distribution the corrections are of moderate size. For the $p_{T, \mathrm{t}}$ distribution we find large corrections for large values of $p_{\mathrm{T}, \mathrm{t}}$. The corrections are almost $50 \%$ for a $p_{\mathrm{T}}$ around $400 \mathrm{GeV}$. As mentioned this could probably be cured by employing a $p_{\mathrm{T}}$-dependent scale.

Figure 3.2 depicts the distributions in the pseudo-rapidity and rapidity of the top quark, $\eta_{\mathrm{t}}$ and $y_{\mathrm{t}}$, and in the rapidity, $y_{\text {jet }}$, of the hard jet. For massless momenta the pseudorapidity, which is defined through

$\eta=-\ln \left(\tan \left(\frac{\vartheta}{2}\right)\right)$

( $\vartheta$ is the scattering angle with respect to the beam axis), is equivalent to the rapidity defined through

$y=\frac{1}{2} \ln \left(\frac{E+p_{z}}{E-p_{z}}\right)$

( $E$ denotes the energy, $p_{z}$ the three-momentum component along the beam axis). For the massive top quark we observe a rather important difference between the $\eta_{\mathrm{t}}$ and $y_{\mathrm{t}}$ distribution. Recently there has been significant interest in the rapidity distribution of the jet, as MC@NLO [81] and Alpgen with MLM matching [82] disagree on this distribution. Our result includes for the first time the full $\mathcal{O}\left(\alpha_{\mathrm{s}}^{4}\right)$ matrix elements. Note that the set-up of [82] differs from the one used 

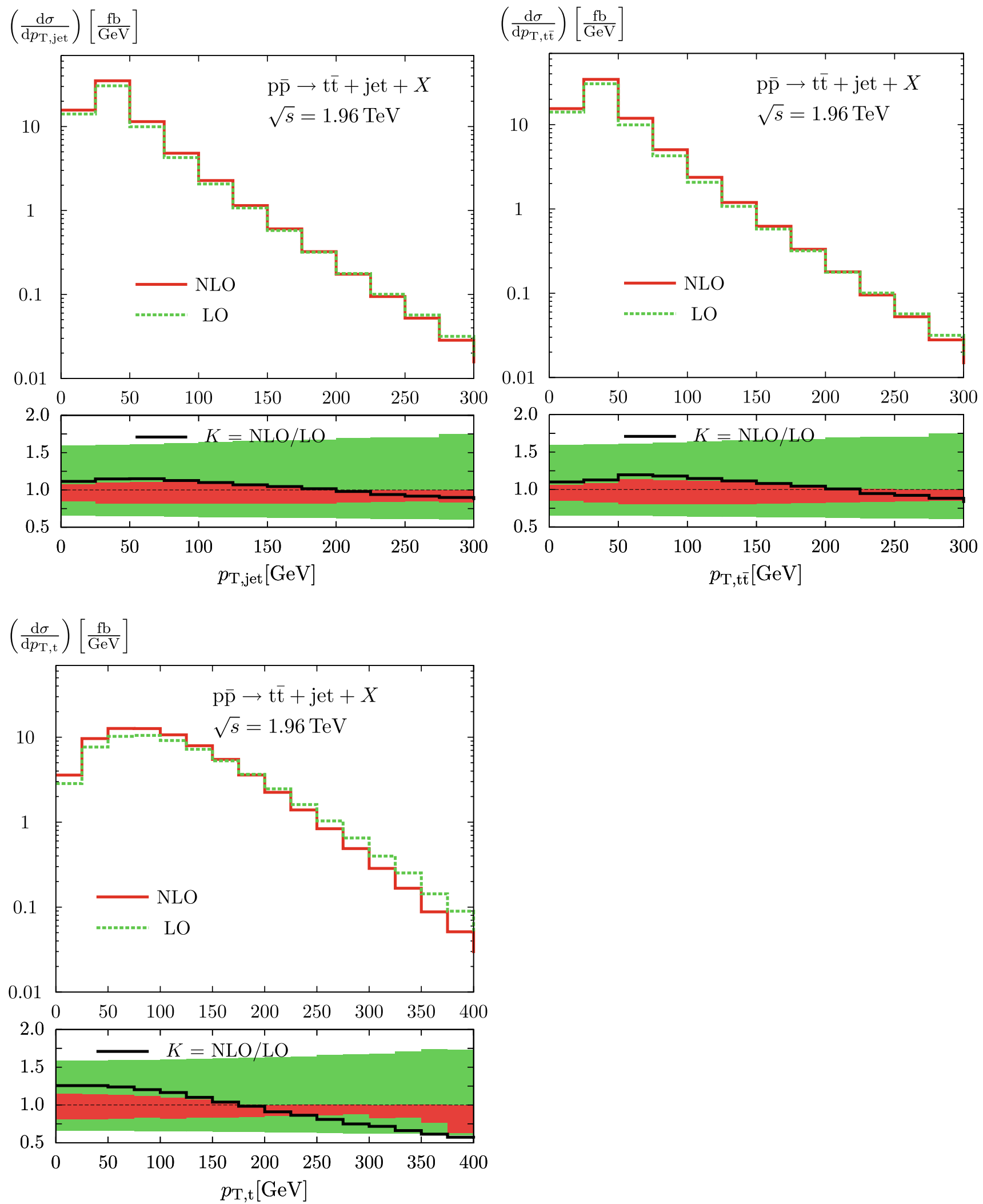

Fig. 3.1 Transverse-momentum distributions of the hard jet $\left(p_{\mathrm{T}, \mathrm{jet}}\right)$, of the total $\bar{t} \bar{t}$ system $\left(p_{\mathrm{T}, \mathrm{tt}}\right)$, and of the top quark $\left(p_{\mathrm{T}, \mathrm{t}}\right)$ at the Tevatron. The lower panels show the ratios $K=\mathrm{NLO} / \mathrm{LO}$ as well as

the LO and NLO scale uncertainties corresponding to a rescaling of $\mu=\mu_{\mathrm{fact}}=\mu_{\mathrm{ren}}=m_{\mathrm{t}}$ by a factor 2 

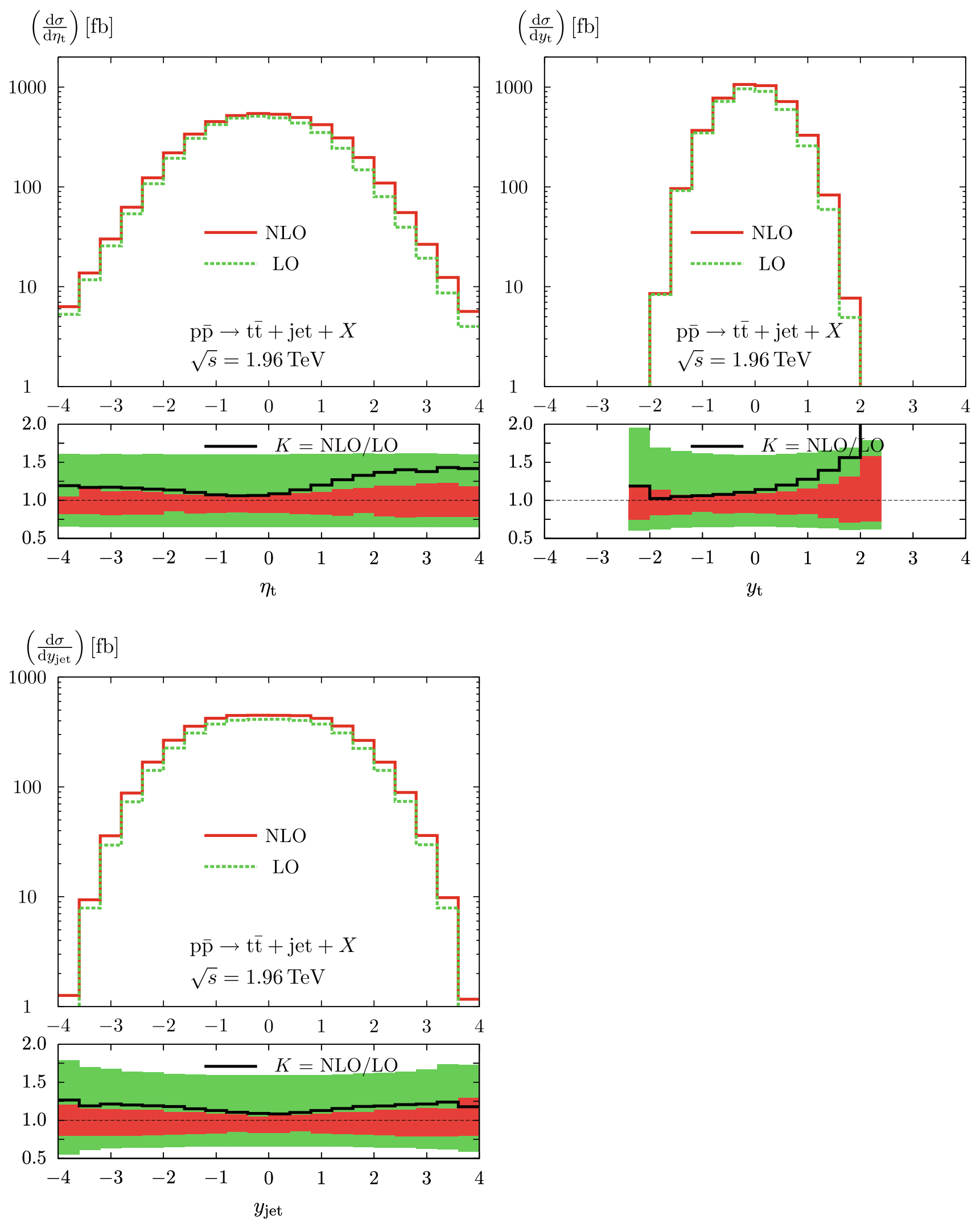

Fig. 3.2 Distributions in the pseudo-rapidity $\left(\eta_{t}\right)$ and rapidity $\left(y_{t}\right)$ of the top quark, and in the rapidity $\left(y_{\text {jet }}\right)$ of the hard jet at the Tevatron. The lower panels show the ratios $K=\mathrm{NLO} / \mathrm{LO}$ as well as

the LO and NLO scale uncertainties corresponding to a rescaling of $\mu=\mu_{\text {fact }}=\mu_{\text {ren }}=m_{\mathrm{t}}$ by a factor 2 
here; therefore the distributions should not be compared directly.

At NLO, 90\% of the events are concentrated within $\left|\eta_{\mathrm{t}}\right|<2.0$. Demanding $\left|y_{\mathrm{t}}\right|<1.2$ selects $96 \%$ of the events. For $y_{\text {jet }}$ we find that $94 \%$ of the events are contained in $\left|y_{\text {jet }}\right|<2.4$.

The reduction of the forward-backward asymmetry $A_{\mathrm{FB}}^{\mathrm{t}}$ discussed above induced by the NLO corrections is clearly visible in the $\eta_{\mathrm{t}}$ and $y_{\mathrm{t}}$ distributions. The corrections are larger in the forward direction. The asymmetry in the LO distributions is thus reduced by the NLO corrections. It is hardly conceivable that this higher-order effect can be absorbed into LO predictions by phase-space-dependent scale choices. It should be realized that the forward-backwardsymmetric rapidity distribution of the hard jet gets distorted by the corrections as well. The corrections increase for large values of $\left|y_{\text {jet }}\right|$.

At least in the regions of the distributions in which the rate is not too much suppressed, the NLO corrections reduce the scale uncertainty of the LO distributions in a similar way as observed for the integrated cross section.

\subsection{Results for the LHC}

Table 3.2 shows the integrated cross section for various values of the cut $p_{\mathrm{T}, \text { jet,cut }}$ on the transverse momentum of the hard tagging jet at the LHC. In contrast to the Tevatron, the gg channel comprises about $70 \%$ of the LO pp cross section, followed by $q \mathrm{~g}$ with about $22 \%$ [37]. We note that the importance of the $q \mathrm{~g}$ channel is very different from the inclusive top-quark production. For inclusive top-quark pair production this channel is suppressed-despite the large parton luminosity in this channel-because it appears only at NLO. For $\bar{t} \bar{t}+1$-jet production the $q \mathrm{~g}$ channel appears already in LO and thus gives a significant contribution due to the large parton luminosity. Comparing the LO and NLO predictions we find again that the large scale dependence of about $100 \%$ in the LO cross section is considerably reduced after including the NLO corrections. The ratio of the NLO $\mathrm{t} \overline{\mathrm{t}}+1$-jet cross section to the total NLO $\bar{t} \overline{\mathrm{t}}$ cross section is

Table 3.2 Cross section $\sigma_{\mathrm{ttj} j \mathrm{t}}$ at the LHC for different values of $p_{\mathrm{T}, \text { jet,cut }}$ for $\mu=\mu_{\mathrm{fact}}=\mu_{\mathrm{ren}}=m_{\mathrm{t}}$. The upper and lower indices are the shifts towards $\mu=m_{\mathrm{t}} / 2$ and $\mu=2 m_{\mathrm{t}}$

\begin{tabular}{lll}
\hline$p_{\text {T,jet,cut }}[\mathrm{GeV}]$ & \multicolumn{1}{c}{$\sigma_{\text {tijjet }}[\mathrm{pb}]$} & NLO \\
\hline 20 & $\mathrm{LO}$ & $692(3) 3_{-62}^{-40}$ \\
50 & $710.8(8)_{-221}^{+358}$ & $376.2(6)_{-48}^{+17}$ \\
100 & $326.6(4)_{-103}^{+168}$ & $175.0(2)_{-24}^{+10}$ \\
200 & $146.7(2)_{-47}^{+77}$ & $52.81(8)_{-6.7}^{+0.8}$ \\
\hline
\end{tabular}

about $47 \%, 22 \%$, and $7 \%$ for a $p_{\mathrm{T}}$ cut of $50 \mathrm{GeV}, 100 \mathrm{GeV}$, and $200 \mathrm{GeV}$, respectively.

In Fig. 3.3 we show the distributions in the transverse momenta of the hard jet, $p_{\mathrm{T}, \mathrm{jet}}$, of the total $\overline{\mathrm{t}} \mathrm{system}, p_{\mathrm{T}, \mathrm{t}}$, and of the top quark, $p_{\mathrm{T}, \mathrm{t}}$. The distributions become harder in $p_{\mathrm{T}}$ when going from the Tevatron to the LHC, as expected from the higher scattering energy. At NLO, 92\% of the events have transverse momenta $p_{\mathrm{T} \text {,jet }}<250 \mathrm{GeV}$, and $94 \%$ have $p_{\mathrm{T}, \mathrm{t}}<300 \mathrm{GeV}$ in the respective distributions. In contrast to the Tevatron, the $p_{\mathrm{T}, \text { jet }}$ and $p_{\mathrm{T}, \mathrm{tt}}$ distributions, which are identical in LO, become different in NLO. For the $p_{\mathrm{T} \text {,jet }}$ distribution the lowest bin $\left(0<p_{\mathrm{T} \text {,jet }}<50 \mathrm{GeV}\right)$ is always empty due to the cut applied. For the $p_{\mathrm{T}, \mathrm{ti}}$ this holds also true in LO, because the transverse momenta between the $\mathrm{t} \overline{\mathrm{t}}$ system and the additional hard jet are balanced. In NLO the lowest bin in the $p_{\mathrm{T}, \mathrm{tt}}$ distribution is populated due to an additional jet. For large $p_{\mathrm{T}}$ the difference between $p_{\mathrm{T}, \mathrm{jet}}$ and $p_{\mathrm{T}, \mathrm{tt}}$ distributions is at the level of about $10 \%$. This is again due to the presence of the additional jet. As observed already for the Tevatron, the shapes of the $p_{\mathrm{T}}$ distributions receive distortions by the corrections.

Figure 3.4 illustrates the distributions in the pseudorapidity and rapidity of the top quark, $\eta_{\mathrm{t}}$ and $y_{\mathrm{t}}$, and in the rapidity, $y_{\text {jet }}$, of the hard jet. In the respective distributions $92 \%$ of the NLO events concentrate within the regions $\left|\eta_{\mathrm{t}}\right|<3.0,96 \%$ have $\left|y_{\mathrm{t}}\right|<2.4$, and $96 \%$ have $\left|y_{\text {jet }}\right|<3.6$ in the respective distributions, i.e. these distributions get broadened roughly by 1 unit in the transition from Tevatron to LHC. As for the Tevatron, we find distortions of the shapes induced by the corrections that are hard to mimic by phasespace-dependent scale choices. All the shown $y$ and $\eta$ distributions at the LHC are forward-backward symmetric, but actually the distributions of top and antitop quarks are intrinsically different. Numerically we do not observe a significant difference, so that we show only the distributions for the top quark.

Again, in all shown distributions a reduction of the scale uncertainty by the NLO corrections is visible that is comparable to the one in the integrated cross section.

\section{Conclusions}

The production of $t \bar{t}+$ jet final states represents important processes both at the Tevatron and the LHC. The signal is interesting in its own right, because large fractions of the

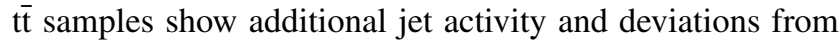
the SM could signal new physics such as top-quark compositeness. Moreover, $\mathrm{t} \overline{\mathrm{t}}+$ jet production delivers a large background to many searches at the LHC, such as for the Higgs boson via weak-vector-boson fusion.

We have presented NLO QCD predictions for $\bar{t} \bar{t}+$ jet production at the Tevatron and the LHC. The NLO corrections reduce the scale uncertainty of the total cross section and of 

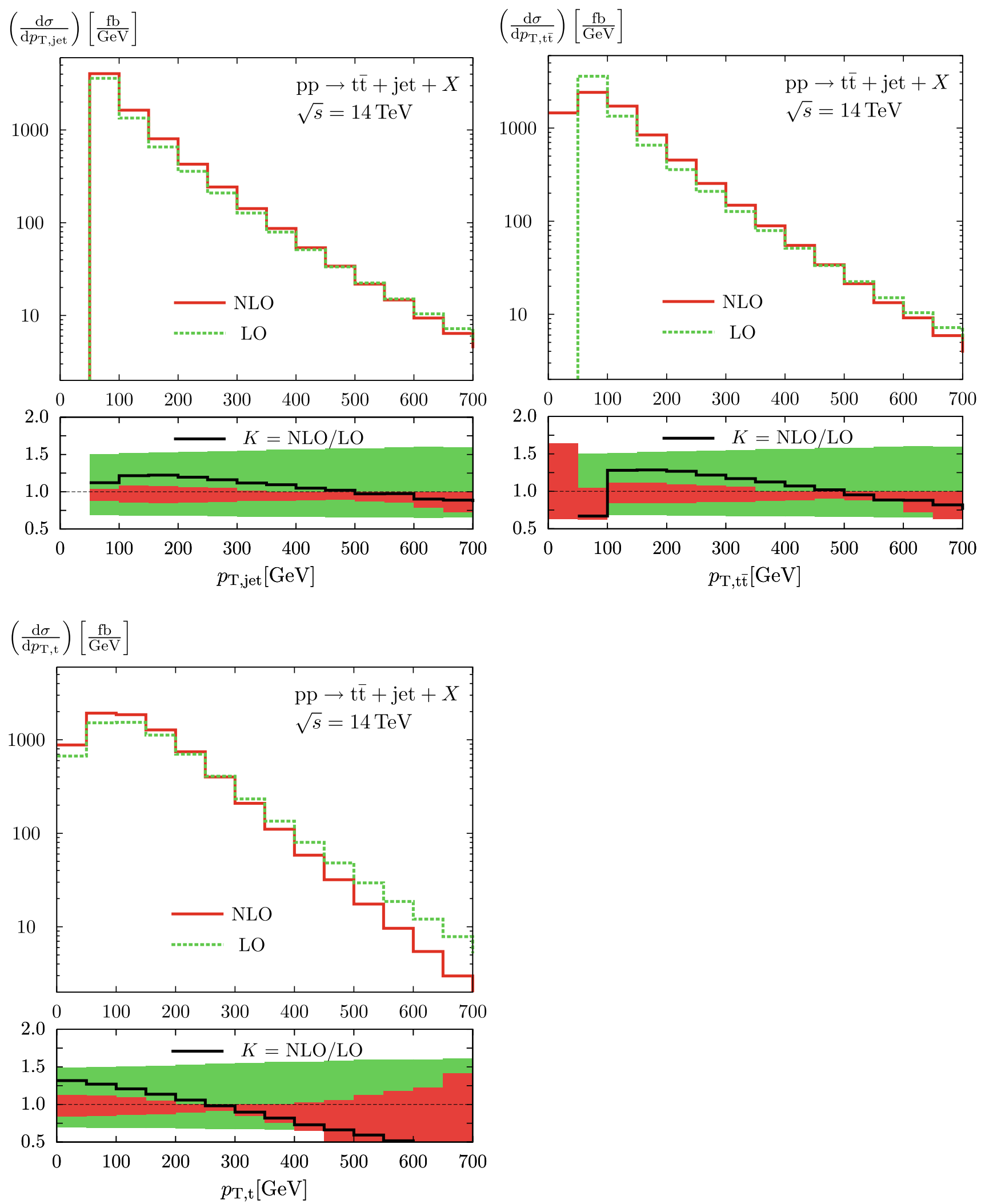

Fig. 3.3 Transverse-momentum distributions of the hard jet ( $\left.p_{\mathrm{T}, \mathrm{jet}}\right)$, of the total $\overline{\mathrm{t}}$ system $\left(p_{\mathrm{T}, \mathrm{t}}\right)$, and of the top quark $\left(p_{\mathrm{T}, \mathrm{t}}\right)$ at the

the LO and NLO scale uncertainties corresponding to a rescaling of LHC. The lower panels show the ratios $K=\mathrm{NLO} / \mathrm{LO}$ as well as $\mu=\mu_{\text {fact }}=\mu_{\text {ren }}=m_{\mathrm{t}}$ by a factor 2 

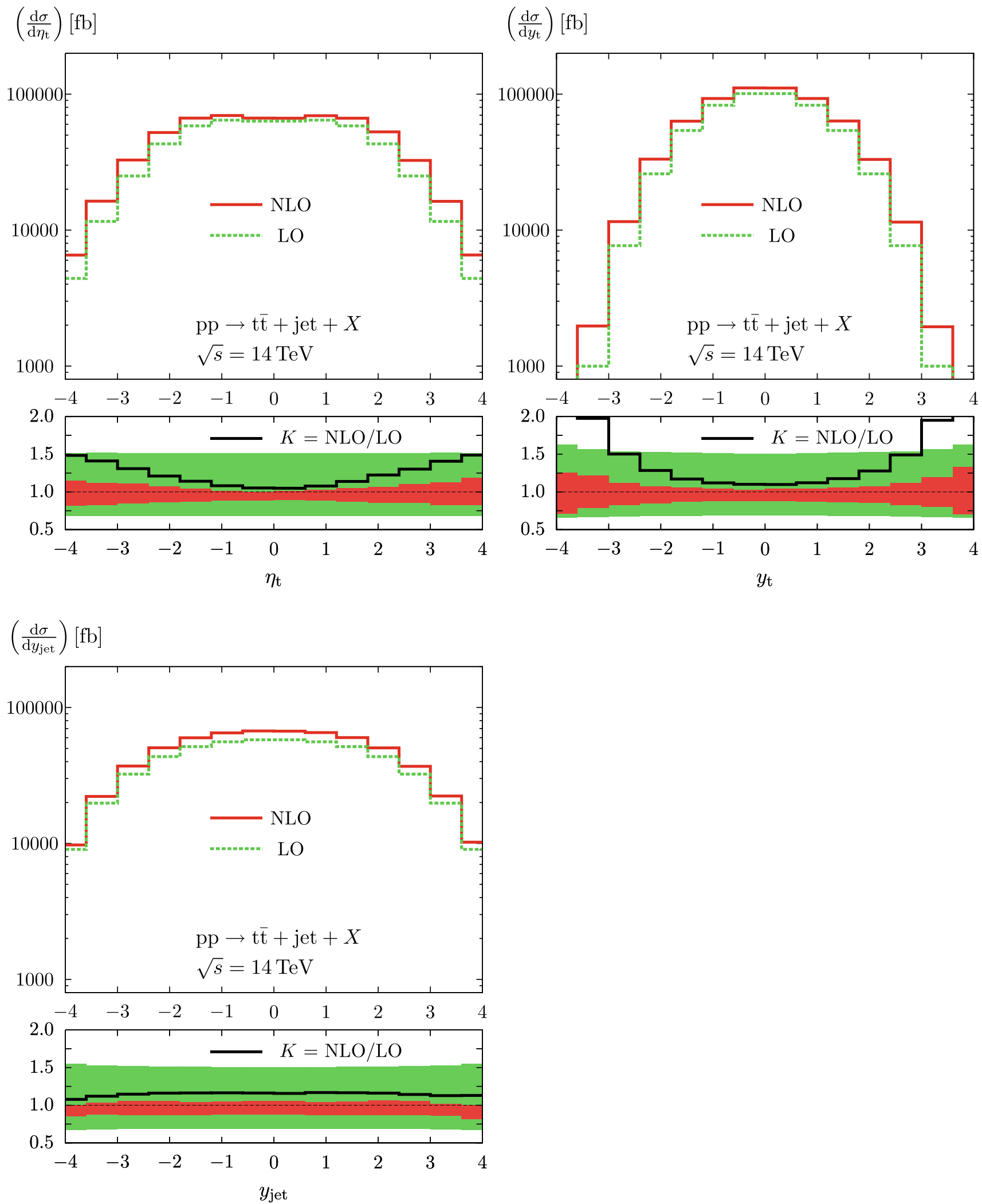

Fig. 3.4 Distributions in the pseudo-rapidity $\left(\eta_{\mathrm{t}}\right)$ and rapidity $\left(y_{\mathrm{t}}\right)$ of the top quark, and in the rapidity $\left(y_{\text {jet }}\right)$ of the hard jet at the LHC. The lower panels show the ratios $K=\mathrm{NLO} / \mathrm{LO}$ as well as

the LO and NLO scale uncertainties corresponding to a rescaling of $\mu=\mu_{\text {fact }}=\mu_{\text {ren }}=m_{\mathrm{t}}$ by a factor 2 
the differential distributions compared to a LO calculation, which can only provide qualitative predictions. Further theoretical improvements could only be achieved by dedicated QCD resummations, since a full treatment at NNLO is certainly out of reach. Already the presented NLO calculation is quite complicated. For this reason we have also documented a set of numerical results for the 1-loop correction and for the real-emission parts at single phase-space points, in order to facilitate comparisons to our calculation by other groups.

The charge asymmetry of the top quark, which is measured at the Tevatron, is significantly decreased at NLO and is almost washed out by the residual scale dependence. We have studied the dependence of the NLO asymmetry on the cut on the transverse momentum of the hard tagging jet. Further refinements in the description of the charge asymmetry are required to stabilize the predictions with respect to higher-order corrections. Moreover, the decay of the top quark should be taken into account properly.

Finally, the presented NLO QCD calculation for the $\overline{\mathrm{t}}+$ jet process represents a building block for a full NNLO QCD prediction for $\bar{t} \bar{t}$ production, a demanding and important calculation that is currently in progress by various groups.

Acknowledgements This work is supported in part by the European Community's Marie-Curie Research Training Network under contract MRTN-CT-2006-035505 "Tools and Precision Calculations for Physics Discoveries at Colliders" and by Deutsche Forschungsgemeinschaft (DFG) through SFB/TR 9. P.U. is financed through a Heisenberg fellowship of DFG.

\section{Appendix A: Benchmark numbers for the virtual corrections}

In order to facilitate a comparison to our calculation, we provide explicit numbers on the squared LO amplitude and the corresponding virtual corrections for a single nonexceptional phase-space point. The set of momenta for $a b \rightarrow \mathrm{t} c$ with the explicit partonic reactions $\mathrm{gg} \rightarrow \mathrm{t \overline {tg }}$, $q \bar{q} \rightarrow \mathrm{ttg}, q \mathrm{~g} \rightarrow \mathrm{tt} q$, and $\mathrm{g} \bar{q} \rightarrow \mathrm{t} \overline{\mathrm{t}} \bar{q}$ is chosen as

$$
\begin{aligned}
p_{a}= & (500,0,0,500), \\
p_{b}= & (500,0,0,-500), \\
p_{\mathrm{t}}= & (458.5331753852783,207.0255169909440,0, \\
& 370.2932732896167), \\
p_{\overline{\mathrm{t}}}= & (206.6000026080000,-10.65693677252589, \\
& 42.52372780926147,-102.3998210421085), \\
p_{c}= & (334.8668220067217,-196.3685802184181, \\
& -42.52372780926147,-267.8934522475083),
\end{aligned}
$$

\begin{tabular}{|c|c|}
\hline \multicolumn{2}{|l|}{$\mathrm{gg} \rightarrow \mathrm{ttg}$} \\
\hline Version 1 & $0.6566843362709776 \times 10^{-3}$ \\
\hline Version 2 & $0.6566843362709785 \times 10^{-3}$ \\
\hline Madgraph & $0.6566843362709775 \times 10^{-3}$ \\
\hline \multicolumn{2}{|l|}{$q \bar{q} \rightarrow \operatorname{ttg}$} \\
\hline Version 1 & $0.5790368001550936 \times 10^{-4}$ \\
\hline Version 2 & $0.5790368001550953 \times 10^{-4}$ \\
\hline Madgraph & $0.5790368001550938 \times 10^{-4}$ \\
\hline \multicolumn{2}{|l|}{$q \mathrm{~g} \rightarrow \overline{\mathrm{tt}} q$} \\
\hline Version 1 & $0.1607845322071585 \times 10^{-4}$ \\
\hline Version 2 & $0.1607845322071587 \times 10^{-4}$ \\
\hline Madgraph & $0.1607845322071585 \times 10^{-4}$ \\
\hline \multicolumn{2}{|l|}{$\mathrm{g} \bar{q} \rightarrow \mathrm{t} \bar{t} \bar{q}$} \\
\hline Version 1 & $0.2603527972645622 \times 10^{-3}$ \\
\hline Version 2 & $0.2603527972645625 \times 10^{-3}$ \\
\hline Madgraph & $0.2603527972645620 \times 10^{-3}$ \\
\hline
\end{tabular}

Table A.1 Color- and spin-averaged LO matrix elements squared

$$
a_{0}\left[\mathrm{GeV}^{-2}\right]
$$

with the obvious notation $p=\left(p^{0}, p^{1}, p^{2}, p^{3}\right)$ and all the components given in $\mathrm{GeV}$. The top-quark mass is set to $m_{\mathrm{t}}=$ $174 \mathrm{GeV}$. We give numbers on the spin- and color-averaged squared LO amplitude $\left|\mathcal{A}_{5}^{(0)}\right|^{2}$ as well as for the contribution $2 \operatorname{Re}\left(\mathcal{A}_{5}^{(0)} \mathcal{A}_{5}^{(1)^{*}}\right)$. For the Born amplitude we factor out the coupling; we define

$$
\frac{1}{4} \frac{1}{\mathcal{N}_{c}} \sum_{\text {spin,color }}\left|\mathcal{A}_{5}^{(0)}\right|^{2}=g_{\mathrm{s}}^{6} a_{0} .
$$

The factor $1 / \mathcal{N}_{c}$ is due to the average over the incoming color. For the channels gg, $q \bar{q}, q \mathrm{~g}$ and $\mathrm{g} \bar{q}$ we have $\mathcal{N}_{c}=64$, 9,24 and 24. Note that the coefficient $a_{0}$ only depends on the chosen phase-space point-which implicitly also contains the information on the top-quark mass. The results for $a_{0}$ are shown in Table A.1. Version 1 and Version 2 correspond to our implementations. For completeness we compare also with Madgraph. The 1-loop contribution $\mathcal{A}^{(1)}$ is renormalized and thus UV finite. However, the virtual corrections still contain collinear and soft singularities. Similar to what has been done in [54] in a comparison of the virtual corrections to $\mathrm{WW}+$ jet production, we use the decomposition

$$
\begin{aligned}
\frac{1}{4} & \frac{1}{\mathcal{N}_{c}} \sum_{\text {spin,color }} 2 \operatorname{Re}\left(\mathcal{A}_{5}^{(0)} \mathcal{A}_{5}^{(1)^{*}}\right) \\
= & g_{\mathrm{s}}^{6} a_{0} \Gamma(1+\epsilon)\left(\frac{4 \pi \mu}{m_{\mathrm{t}}^{2}}\right)^{\epsilon} \\
& \times\left(c_{-2} \frac{1}{\epsilon^{2}}+c_{-1} \frac{1}{\epsilon}+c_{0}+\mathcal{O}(\epsilon)\right) .
\end{aligned}
$$


Table A.2 Coefficients for color- and spin-averaged virtual corrections
Table A.3 Coefficients for color- and spin-averaged results for the I-operator

\begin{tabular}{|c|c|c|c|}
\hline & $c_{-2}$ & $c_{-1}$ & $c_{0}$ \\
\hline \multicolumn{4}{|l|}{$\mathrm{gg} \rightarrow \mathrm{t} \overline{\mathrm{tg}}$} \\
\hline Version 1 & -0.1540118420981379 & 0.0731096895036588 & 0.5295183452346090 \\
\hline Version 2 & -0.1540118421074573 & 0.0731096894943437 & 0.5295183452413002 \\
\hline \multicolumn{4}{|l|}{$q \bar{q} \rightarrow \operatorname{ttg}$} \\
\hline Version 1 & -0.0969704191047176 & -0.0126983208241891 & 0.2435672439083931 \\
\hline Version 2 & -0.0969704191046950 & -0.0126983208241662 & 0.2435672439081981 \\
\hline \multicolumn{4}{|l|}{$q \mathrm{~g} \rightarrow \mathrm{t} q$} \\
\hline Version 1 & -0.0969704191047088 & -0.0056430956994203 & 0.4003849386477017 \\
\hline Version 2 & -0.0969704191046951 & -0.0056430956994064 & 0.4003849386472126 \\
\hline \multicolumn{4}{|l|}{$\mathrm{g} \bar{q} \rightarrow \mathrm{t} \bar{q} \bar{q}$} \\
\hline Version 1 & -0.0969704191046802 & 0.0833362739128030 & 0.5384721403213878 \\
\hline Version 2 & -0.0969704191046950 & 0.0833362739127883 & 0.5384721403213897 \\
\hline & $c_{-2}$ & $c_{-1}$ & $c_{0}$ \\
\hline \multicolumn{4}{|l|}{$\mathrm{gg} \rightarrow \overline{\mathrm{ttg}}$} \\
\hline Version 1 & 0.1540118421074569 & -0.0731096894943435 & -0.5280576886301999 \\
\hline Version 2 & 0.1540118421074573 & -0.0731096894943437 & -0.5280576886302015 \\
\hline \multicolumn{4}{|l|}{$q \bar{q} \rightarrow \operatorname{ttg}$} \\
\hline Version 1 & 0.0969704191046952 & 0.0126983208241661 & -0.3992776407671517 \\
\hline Version 2 & 0.0969704191046950 & 0.0126983208241662 & -0.3992776407671513 \\
\hline \multicolumn{4}{|l|}{$q \mathrm{~g} \rightarrow \mathrm{t} \overline{\mathrm{t}} q$} \\
\hline Version 1 & 0.0969704191046950 & 0.0056430956994063 & -0.4069645466913195 \\
\hline Version 2 & 0.0969704191046951 & 0.0056430956994064 & -0.4069645466913194 \\
\hline \multicolumn{4}{|l|}{$\mathrm{g} \bar{q} \rightarrow \mathrm{t} \bar{q} \bar{q}$} \\
\hline Version 1 & 0.0969704191046950 & -0.0833362739127882 & -0.3392937280293060 \\
\hline Version2 & 0.0969704191046950 & -0.0833362739127883 & -0.3392937280293059 \\
\hline
\end{tabular}

The results are shown in Table A.2. In addition we also give the corresponding results for the I-operator of the dipole subtraction function as defined in [75], with the auxiliary parameter $\kappa=2 / 3$. We us the same decomposition as for the 1 -loop corrections. The individual coefficients are shown in Table A.3. Note that the coefficients $c_{i}$ with $i=-2,-1,0$ contain 1 factor of $\alpha_{\mathrm{s}}$. We use

$$
\begin{aligned}
\alpha_{s}(\mu) & =\frac{4 \pi}{\beta_{0} L}\left(1-\frac{\beta_{1}}{\beta_{0}^{2}} \frac{\ln L}{L}\right), \\
L & =\ln \left(\mu^{2} / \Lambda^{2}\right), \quad \beta_{0}=11-\frac{2}{3} N_{f}, \\
\beta_{1} & =102-\frac{38}{3} N_{f} .
\end{aligned}
$$

with $N_{f}=5$ and $\Lambda=226 \mathrm{MeV}$, leading to

$\alpha_{\mathrm{S}}\left(m_{\mathrm{t}}\right)=0.1075205492734706$.
For the LO amplitudes we find an agreement of at least 14 digits - pretty close to what one can get using 64 bit double precision with 53 bits for the mantissa. ${ }^{3}$ For the 1-loop corrections we find at least an agreement of 10 digits for the finite terms. For the $q \bar{q}, q \mathrm{~g}$ and $\mathrm{g} \bar{q}$ channels, which are numerically less involved, we find an agreement of up to 14 digits. The finite terms from the I-operator agree even better. The coefficients of all divergences typically agree to 10 digits, or better in the cases with external quarks. We note, however, that we do not cancel the IR divergences numerically. We also observe a cancellation between the finite

\footnotetext{
${ }^{3}$ Note that some of the code was run on a x86-64 architecture, where usually floating-point arithmetic is typically done in the SMD unit of the processor and thus restricted to 64 bit also for intermediate results. (On x86 architectures arithmetic is usually done in the FPU which usually works with extended precision for intermediate results (80 bit). The precision is reduced when results are stored back to memory.)
} 
part of the I-operator and the corresponding virtual contributions. For the $\mathrm{gg} \rightarrow \mathrm{t} \overline{\mathrm{tg}}$ channel almost three digits are cancelled in the combination.

\section{Appendix B: Benchmark numbers for the subtraction terms}

In this section we also give results for the subtraction term in the dipole formalism for 1 phase-space point. We find this useful to facilitate the comparison of upcoming calculations. Recently some effort has been invested to automatize this part of the calculation [83-86]. The results presented here may provide an interesting benchmark point for these attempts. The set of momenta for $a b \rightarrow \mathrm{tt} c d$ is chosen as

$$
\begin{aligned}
p_{a}= & (2100,-0,-0,2100), \\
p_{b}= & (2800,-0,-0,-2800), \\
p_{\mathrm{t}}= & (1581.118367308447,1254.462316247655, \\
& -766.9360998604944,-554.7905976902205), \\
p_{\overline{\mathrm{t}}}= & (1460.449317799282,-975.9731477430979, \\
& -466.5314749495881,965.6402060944737), \\
p_{c}= & (545.4084744819,218.7220720302516, \\
& 472.0439121434804,-163.7241712507502), \\
p_{d}= & (1313.023840410371,-497.2112405348086, \\
& 761.423662666602,-947.1254371535031),
\end{aligned}
$$

with all components given in $\mathrm{GeV}$. The top-quark mass is set to $m_{\mathrm{t}}=174 \mathrm{GeV}$. We give numbers on the spin- and coloraveraged squared real-emission amplitude $\left|\mathcal{A}_{6}^{(0)}\right|^{2}$ as well as for the sum of the subtraction terms. We define the numbers $b_{0}$ by

$\frac{1}{4} \frac{1}{\mathcal{N}_{c}} \frac{1}{\mathcal{S}} \sum_{\text {spin,color }}\left|\mathcal{A}_{6}^{(0)}\right|^{2}=b_{0}$.

The factor $1 / 4$ accounts for the average over the spins of the initial partons, the factor $1 / \mathcal{N}_{c}$ is due to the average over the color of the incoming partons. $\mathcal{N}_{c}$ contains a factor 8 for every incoming gluon and a factor 3 for every incoming quark or antiquark. $\mathcal{S}$ is the symmetry factor accounting for identical particles in the final state. The numbers for the dipole subtraction terms are defined analogously:

$$
\frac{1}{4} \frac{1}{\mathcal{S}} \sum_{\text {pairs } i, j} \sum_{k \neq i, j} \mathcal{D}_{i j, k}=d_{0}
$$

Note that the factor for the average over the color of the incoming partons is included in the definition of $\mathcal{D}_{i j, k}$. The numbers $b_{0}$ and $d_{0}$ contain the strong coupling constant. As numerical value for $\alpha_{\mathrm{s}}$ we use again (A.4) with $N_{f}=5$ and $\Lambda=226 \mathrm{MeV}$, i.e. the value given in (A.5). The results for

\begin{tabular}{|c|c|c|}
\hline & $b_{0}\left[\mathrm{GeV}^{-4}\right]$ & $d_{0}\left[\mathrm{GeV}^{-4}\right]$ \\
\hline \multicolumn{3}{|c|}{$\mathrm{g}\left(p_{a}\right) \mathrm{g}\left(p_{b}\right) \rightarrow \mathrm{t}\left(p_{\mathrm{t}}\right) \overline{\mathrm{t}}\left(p_{\overline{\mathrm{t}}}\right) \mathrm{g}\left(p_{c}\right) \mathrm{g}\left(p_{d}\right)$} \\
\hline Version 1 & $3.12815868347843 \times 10^{-9}$ & $4.1037601540955 \times 10^{-9}$ \\
\hline Version 2 & $3.12815868347842 \times 10^{-9}$ & $4.1037601540962 \times 10^{-9}$ \\
\hline \multicolumn{3}{|c|}{$q\left(p_{a}\right) \bar{q}\left(p_{b}\right) \rightarrow \mathrm{t}\left(p_{\mathrm{t}}\right) \overline{\mathrm{t}}\left(p_{\overline{\mathrm{t}}}\right) \mathrm{g}\left(p_{c}\right) \mathrm{g}\left(p_{d}\right)$} \\
\hline Version 1 & $4.48308845446477 \times 10^{-10}$ & $4.90476067759639 \times 10^{-10}$ \\
\hline Version 2 & $4.48308845446475 \times 10^{-10}$ & $4.90476067759631 \times 10^{-10}$ \\
\hline \multicolumn{3}{|c|}{$q\left(p_{a}\right) \mathrm{g}\left(p_{b}\right) \rightarrow \mathrm{t}\left(p_{\mathrm{t}}\right) \overline{\mathrm{t}}\left(p_{\overline{\mathrm{t}}}\right) \mathrm{g}\left(p_{c}\right) q\left(p_{d}\right)$} \\
\hline Version 1 & $1.10256509258713 \times 10^{-10}$ & $1.919073353538 \times 10^{-10}$ \\
\hline Version 2 & $1.10256509258713 \times 10^{-10}$ & $1.919073353539 \times 10^{-10}$ \\
\hline \multicolumn{3}{|c|}{$\bar{q}\left(p_{a}\right) \mathrm{g}\left(p_{b}\right) \rightarrow \mathrm{t}\left(p_{\mathrm{t}}\right) \overline{\mathrm{t}}\left(p_{\overline{\mathrm{t}}}\right) \bar{q}\left(p_{c}\right) \mathrm{g}\left(p_{d}\right)$} \\
\hline Version 1 & $1.384600673183816 \times 10^{-10}$ & $3.3382231835799 \times 10^{-10}$ \\
\hline Version 2 & $1.384600673183812 \times 10^{-10}$ & $3.3382231835798 \times 10^{-10}$ \\
\hline \multicolumn{3}{|c|}{$\mathrm{g}\left(p_{a}\right) \mathrm{g}\left(p_{b}\right) \rightarrow \mathrm{t}\left(p_{\mathrm{t}}\right) \overline{\mathrm{t}}\left(p_{\overline{\mathrm{t}}}\right) \bar{q}\left(p_{c}\right) q\left(p_{d}\right)$} \\
\hline Version 1 & $2.42841040229558 \times 10^{-10}$ & $4.271065781530 \times 10^{-10}$ \\
\hline Version 2 & $2.42841040229557 \times 10^{-10}$ & $4.271065781532 \times 10^{-10}$ \\
\hline
\end{tabular}
$b_{0}$ and $d_{0}$ are shown in Tables B.1 and B.2. The two implementations agree at least to 14 digits for the matrix elements squared and at least to 12 digits for the sum of the subtraction terms.
Table B.1 Color- and spin-averaged real-emission matrix element squared and dipole subtraction terms related to the processes $0 \rightarrow$ ttgggg and $0 \rightarrow \mathrm{t} \overline{\mathrm{t}} q \bar{q} \mathrm{gg}$ 
Table B.2 Color- and spin-averaged real-emission matrix element squared and dipole subtraction terms related to the processes $0 \rightarrow \operatorname{tt} q \bar{q} q^{\prime} \bar{q}^{\prime}$ and $0 \rightarrow \overline{\mathrm{t}} q \bar{q} q \bar{q}$

\begin{tabular}{|c|c|c|}
\hline & $b_{0}\left[\mathrm{GeV}^{-4}\right]$ & $d_{0}\left[\mathrm{GeV}^{-4}\right]$ \\
\hline \multicolumn{3}{|c|}{$q\left(p_{a}\right) q^{\prime}\left(p_{b}\right) \rightarrow \mathrm{t}\left(p_{\mathrm{t}}\right) \overline{\mathrm{t}}\left(p_{\overline{\mathrm{t}}}\right) q^{\prime}\left(p_{c}\right) q\left(p_{d}\right)$} \\
\hline Version 1 & $4.44137855516180 \times 10^{-12}$ & $1.6381811832266 \times 10^{-11}$ \\
\hline Version 2 & $4.44137855516180 \times 10^{-12}$ & $1.6381811832275 \times 10^{-11}$ \\
\hline \multicolumn{3}{|c|}{$\bar{q}\left(p_{a}\right) \bar{q}^{\prime}\left(p_{b}\right) \rightarrow \mathrm{t}\left(p_{\mathrm{t}}\right) \overline{\mathrm{t}}\left(p_{\overline{\mathrm{t}}}\right) \bar{q}\left(p_{c}\right) \bar{q}^{\prime}\left(p_{d}\right)$} \\
\hline Version 1 & $1.733763330485899 \times 10^{-11}$ & $1.06832579841007 \times 10^{-10}$ \\
\hline Version 2 & $1.733763330485899 \times 10^{-11}$ & $1.06832579841000 \times 10^{-10}$ \\
\hline \multicolumn{3}{|c|}{$q\left(p_{a}\right) \bar{q}^{\prime}\left(p_{b}\right) \rightarrow \mathrm{t}\left(p_{\mathrm{t}}\right) \overline{\mathrm{t}}\left(p_{\overline{\mathrm{t}}}\right) \bar{q}^{\prime}\left(p_{c}\right) q\left(p_{d}\right)$} \\
\hline Version 1 & $4.796260245409952 \times 10^{-12}$ & $1.8776008214791 \times 10^{-11}$ \\
\hline Version 2 & $4.796260245409957 \times 10^{-12}$ & $1.8776008214799 \times 10^{-11}$ \\
\hline \multicolumn{3}{|c|}{$q\left(p_{a}\right) \bar{q}\left(p_{b}\right) \rightarrow \mathrm{t}\left(p_{\mathrm{t}}\right) \overline{\mathrm{t}}\left(p_{\overline{\mathrm{t}}}\right) \bar{q}^{\prime}\left(p_{c}\right) q^{\prime}\left(p_{d}\right)$} \\
\hline Version 1 & $6.13924303047741 \times 10^{-11}$ & $6.990891152615 \times 10^{-11}$ \\
\hline Version 2 & $6.13924303047739 \times 10^{-11}$ & $6.990891152614 \times 10^{-11}$ \\
\hline \multicolumn{3}{|c|}{$q\left(p_{a}\right) q\left(p_{b}\right) \rightarrow \mathrm{t}\left(p_{\mathrm{t}}\right) \overline{\mathrm{t}}\left(p_{\overline{\mathrm{t}}}\right) q\left(p_{c}\right) q\left(p_{d}\right)$} \\
\hline Version 1 & $1.371477814148721 \times 10^{-11}$ & $4.1848434402744 \times 10^{-11}$ \\
\hline Version 2 & $1.371477814148719 \times 10^{-11}$ & $4.1848434402750 \times 10^{-11}$ \\
\hline \multicolumn{3}{|c|}{$\bar{q}\left(p_{a}\right) \bar{q}\left(p_{b}\right) \rightarrow \mathrm{t}\left(p_{\mathrm{t}}\right) \overline{\mathrm{t}}\left(p_{\overline{\mathrm{t}}}\right) \bar{q}\left(p_{c}\right) \bar{q}\left(p_{d}\right)$} \\
\hline Version 1 & $1.411042000289490 \times 10^{-11}$ & $6.3674516988854 \times 10^{-11}$ \\
\hline Version 2 & $1.411042000289488 \times 10^{-11}$ & $6.3674516988857 \times 10^{-11}$ \\
\hline \multicolumn{3}{|c|}{$q\left(p_{a}\right) \bar{q}\left(p_{b}\right) \rightarrow \mathrm{t}\left(p_{\mathrm{t}}\right) \overline{\mathrm{t}}\left(p_{\overline{\mathrm{t}}}\right) \bar{q}\left(p_{c}\right) q\left(p_{d}\right)$} \\
\hline Version 1 & $2.054843839960259 \times 10^{-11}$ & $3.6253236096328 \times 10^{-11}$ \\
\hline Version 2 & $2.054843839960252 \times 10^{-11}$ & $3.6253236096334 \times 10^{-11}$ \\
\hline
\end{tabular}

Table C.1 The transverse-momentum distribution of the hard jet at the Tevatron

\begin{tabular}{llll}
\hline$p_{\mathrm{T}, \text { jet }}[\mathrm{GeV}]$ & $\frac{\mathrm{d} \sigma}{\mathrm{d} p_{\mathrm{T}, \mathrm{jet}}}\left[\frac{\mathrm{fb}}{\mathrm{GeV}}\right]$ & & \\
\cline { 2 - 4 } & $\mu=m_{\mathrm{t}} / 2$ & $\mu=m_{\mathrm{t}}$ & $\mu=2 m_{\mathrm{t}}$ \\
\hline 12.5 & $16.83 \pm 0.07$ & $15.70 \pm 0.04$ & $13.4 \pm 0.2$ \\
37.5 & $38.47 \pm 0.04$ & $35.08 \pm 0.03$ & $28.83 \pm 0.02$ \\
62.5 & $12.67 \pm 0.02$ & $11.45 \pm 0.01$ & $9.340 \pm 0.009$ \\
87.5 & $5.29 \pm 0.01$ & $4.805 \pm 0.008$ & $3.92 \pm 0.005$ \\
112.5 & $2.465 \pm 0.007$ & $2.277 \pm 0.006$ & $1.864 \pm 0.003$ \\
137.5 & $1.216 \pm 0.005$ & $1.146 \pm 0.003$ & $0.943 \pm 0.002$ \\
162.5 & $0.629 \pm 0.004$ & $0.604 \pm 0.002$ & $0.496 \pm 0.001$ \\
187.5 & $0.326 \pm 0.003$ & $0.324 \pm 0.002$ & $0.2659 \pm 0.0007$ \\
212.5 & $0.173 \pm 0.002$ & $0.174 \pm 0.001$ & $0.1460 \pm 0.0005$ \\
237.5 & $0.093 \pm 0.001$ & $0.0945 \pm 0.0008$ & $0.0796 \pm 0.0004$ \\
262.5 & $0.047 \pm 0.001$ & $0.0522 \pm 0.0006$ & $0.0445 \pm 0.0003$ \\
287.5 & $0.0252 \pm 0.0007$ & $0.0285 \pm 0.0003$ & $0.0238 \pm 0.0002$ \\
312.5 & $0.0113 \pm 0.0006$ & $0.0151 \pm 0.0003$ & $0.0129 \pm 0.0001$ \\
\hline
\end{tabular}

Table C.2 The transverse-momentum distribution of the total $\bar{t}$ system at the Tevatron

\begin{tabular}{llll}
\hline$p_{\mathrm{T}, \mathrm{tt}}[\mathrm{GeV}]$ & $\frac{\mathrm{d} \sigma}{\mathrm{d} p_{\mathrm{T}, \mathrm{tt}}}\left[\frac{\mathrm{fb}}{\mathrm{GeV}}\right]$ & & \\
& $\mu=m_{\mathrm{t}} / 2$ & $\mu=m_{\mathrm{t}}$ & $\mu=2 m_{\mathrm{t}}$ \\
\hline 12.5 & $16.43 \pm 0.07$ & $15.49 \pm 0.04$ & $13.2 \pm 0.2$ \\
37.5 & $37.25 \pm 0.05$ & $34.43 \pm 0.03$ & $28.46 \pm 0.02$ \\
62.5 & $13.56 \pm 0.02$ & $11.91 \pm 0.01$ & $9.61 \pm 0.01$ \\
87.5 & $5.66 \pm 0.01$ & $5.036 \pm 0.007$ & $4.053 \pm 0.004$ \\
112.5 & $2.648 \pm 0.007$ & $2.373 \pm 0.005$ & $1.919 \pm 0.003$ \\
137.5 & $1.309 \pm 0.006$ & $1.194 \pm 0.003$ & $0.967 \pm 0.002$ \\
162.5 & $0.669 \pm 0.004$ & $0.623 \pm 0.002$ & $0.509 \pm 0.001$ \\
187.5 & $0.345 \pm 0.002$ & $0.332 \pm 0.001$ & $0.2720 \pm 0.0007$ \\
212.5 & $0.180 \pm 0.002$ & $0.1791 \pm 0.0008$ & $0.1482 \pm 0.0005$ \\
237.5 & $0.096 \pm 0.001$ & $0.0953 \pm 0.0006$ & $0.0797 \pm 0.0004$ \\
262.5 & $0.0466 \pm 0.0009$ & $0.0525 \pm 0.0005$ & $0.0443 \pm 0.0002$ \\
287.5 & $0.0243 \pm 0.0007$ & $0.0280 \pm 0.0003$ & $0.0239 \pm 0.0002$ \\
312.5 & $0.0113 \pm 0.0005$ & $0.0144 \pm 0.0003$ & $0.0126 \pm 0.0001$ \\
\hline
\end{tabular}


Table C.3 The transverse-momentum distribution of the top quark at the Tevatron

\begin{tabular}{lccc}
\hline$p_{\mathrm{T}, \mathrm{t}}[\mathrm{GeV}]$ & $\frac{\mathrm{d} \sigma}{\mathrm{d} p_{\mathrm{T}, \mathrm{t}}}\left[\frac{\mathrm{fb}}{\mathrm{GeV}}\right]$ & & \\
& $\mu=m_{\mathrm{t}} / 2$ & $\mu=m_{\mathrm{t}}$ & $\mu=2 m_{\mathrm{t}}$ \\
\hline 12.5 & $4.12 \pm 0.02$ & $3.58 \pm 0.01$ & $2.910 \pm 0.007$ \\
37.5 & $10.96 \pm 0.03$ & $9.62 \pm 0.02$ & $7.78 \pm 0.02$ \\
62.5 & $14.31 \pm 0.03$ & $12.68 \pm 0.02$ & $10.35 \pm 0.02$ \\
87.5 & $14.11 \pm 0.03$ & $12.63 \pm 0.02$ & $10.58 \pm 0.02$ \\
112.5 & $11.67 \pm 0.03$ & $10.66 \pm 0.02$ & $8.72 \pm 0.01$ \\
137.5 & $8.53 \pm 0.02$ & $7.94 \pm 0.01$ & $6.59 \pm 0.01$ \\
162.5 & $5.75 \pm 0.02$ & $5.48 \pm 0.01$ & $4.570 \pm 0.008$ \\
187.5 & $3.59 \pm 0.03$ & $3.59 \pm 0.01$ & $3.019 \pm 0.008$ \\
212.5 & $2.23 \pm 0.02$ & $2.24 \pm 0.01$ & $1.923 \pm 0.006$ \\
237.5 & $1.30 \pm 0.01$ & $1.391 \pm 0.008$ & $1.189 \pm 0.004$ \\
262.5 & $0.76 \pm 0.01$ & $0.837 \pm 0.006$ & $0.721 \pm 0.004$ \\
287.5 & $0.43 \pm 0.01$ & $0.488 \pm 0.004$ & $0.428 \pm 0.003$ \\
312.5 & $0.237 \pm 0.009$ & $0.286 \pm 0.004$ & $0.254 \pm 0.003$ \\
337.5 & $0.139 \pm 0.006$ & $0.167 \pm 0.003$ & $0.146 \pm 0.002$ \\
362.5 & $0.067 \pm 0.005$ & $0.088 \pm 0.003$ & $0.082 \pm 0.002$ \\
387.5 & $0.032 \pm 0.005$ & $0.051 \pm 0.002$ & $0.047 \pm 0.001$ \\
\hline & & &
\end{tabular}

Table C.4 The pseudo-rapidity distribution of the top quark at the Tevatron

\begin{tabular}{|c|c|c|c|}
\hline \multirow[t]{2}{*}{$\eta_{\mathrm{t}}$} & \multicolumn{3}{|l|}{$\frac{\mathrm{d} \sigma}{\mathrm{d} \eta_{\mathrm{t}}}[\mathrm{fb}]$} \\
\hline & $\mu=m_{\mathrm{t}} / 2$ & $\mu=m_{\mathrm{t}}$ & $\mu=2 m_{\mathrm{t}}$ \\
\hline-3.8 & $6.6 \pm 0.2$ & $6.3 \pm 0.1$ & $5.2 \pm 0.1$ \\
\hline-3.4 & $16.0 \pm 0.3$ & $13.7 \pm 0.2$ & $11.2 \pm 0.1$ \\
\hline-3.0 & $33.5 \pm 0.4$ & $30.1 \pm 0.3$ & $24.1 \pm 0.2$ \\
\hline-2.6 & $70.1 \pm 0.8$ & $62.5 \pm 0.3$ & $50.9 \pm 0.2$ \\
\hline-2.2 & $136.4 \pm 0.8$ & $123.3 \pm 0.5$ & $100.3 \pm 0.3$ \\
\hline-1.8 & $237 \pm 1$ & $219.6 \pm 0.9$ & $188 \pm 9$ \\
\hline-1.4 & $363 \pm 1$ & $338.1 \pm 0.8$ & $279.6 \pm 0.5$ \\
\hline-1.0 & $483 \pm 1$ & $451.8 \pm 0.8$ & $376.2 \pm 0.6$ \\
\hline-0.6 & $546 \pm 2$ & $519 \pm 1$ & $437 \pm 2$ \\
\hline-0.2 & $573 \pm 2$ & $543.8 \pm 0.9$ & $454.5 \pm 0.6$ \\
\hline 0.2 & $573 \pm 2$ & $534.3 \pm 0.8$ & $446.2 \pm 0.6$ \\
\hline 0.6 & $542 \pm 1$ & $496.6 \pm 0.9$ & $409.1 \pm 0.6$ \\
\hline 1.0 & $467 \pm 1$ & $420.7 \pm 0.8$ & $341.4 \pm 0.6$ \\
\hline 1.4 & $354 \pm 1$ & $310 \pm 1$ & $248.6 \pm 0.5$ \\
\hline 1.8 & $229 \pm 1$ & $197.2 \pm 0.5$ & $165 \pm 9$ \\
\hline 2.2 & $130.5 \pm 0.7$ & $109.4 \pm 0.4$ & $86.4 \pm 0.3$ \\
\hline 2.6 & $65.9 \pm 0.5$ & $55.3 \pm 0.3$ & $43.0 \pm 0.2$ \\
\hline 3.0 & $32.4 \pm 0.4$ & $26.6 \pm 0.2$ & $20.9 \pm 0.2$ \\
\hline 3.4 & $15.2 \pm 0.3$ & $12.4 \pm 0.1$ & $9.7 \pm 0.1$ \\
\hline 3.8 & $6.7 \pm 0.2$ & $5.7 \pm 0.1$ & $4.42 \pm 0.08$ \\
\hline
\end{tabular}

Table C.5 The rapidity distribution of the top quark at the Tevatron

\begin{tabular}{lccc}
\hline$y_{\mathrm{t}}$ & $\frac{\mathrm{d} \sigma}{\mathrm{d} y_{\mathrm{t}}}[\mathrm{fb}]$ & & \\
\cline { 2 - 4 } & $\mu=m_{\mathrm{t}} / 2$ & $\mu=m_{\mathrm{t}}$ & $\mu=2 m_{\mathrm{t}}$ \\
\hline-1.8 & $9.8 \pm 0.3$ & $8.5 \pm 0.1$ & $6.9 \pm 0.1$ \\
-1.4 & $103.5 \pm 0.8$ & $96.3 \pm 0.4$ & $78.5 \pm 0.3$ \\
-1.0 & $388 \pm 1$ & $368.4 \pm 0.8$ & $314 \pm 9$ \\
-0.6 & $826 \pm 2$ & $777 \pm 2$ & $645.0 \pm 0.8$ \\
-0.2 & $1144 \pm 2$ & $1063 \pm 1$ & $888 \pm 2$ \\
0.2 & $1131 \pm 3$ & $1035 \pm 1$ & $856.6 \pm 0.8$ \\
0.6 & $799 \pm 2$ & $716 \pm 1$ & $581.9 \pm 0.8$ \\
1.0 & $379 \pm 1$ & $329.4 \pm 0.9$ & $270 \pm 9$ \\
1.4 & $100.9 \pm 0.6$ & $82.9 \pm 0.3$ & $63.5 \pm 0.2$ \\
1.8 & $10.1 \pm 0.1$ & $7.68 \pm 0.08$ & $5.46 \pm 0.07$ \\
\hline
\end{tabular}

Table C.6 The rapidity distribution of the hard jet at the Tevatron

\begin{tabular}{|c|c|c|c|}
\hline \multirow[t]{2}{*}{$y_{\text {jet }}$} & \multicolumn{3}{|l|}{$\frac{\mathrm{d} \sigma}{\mathrm{d} y_{\mathrm{jet}}}[\mathrm{fb}]$} \\
\hline & $\mu=m_{\mathrm{t}} / 2$ & $\mu=m_{\mathrm{t}}$ & $\mu=2 m_{\mathrm{t}}$ \\
\hline-3.8 & $1.5 \pm 0.1$ & $1.26 \pm 0.09$ & $1.0 \pm 0.1$ \\
\hline-3.4 & $10.8 \pm 0.4$ & $9.4 \pm 0.2$ & $7.5 \pm 0.2$ \\
\hline-3.0 & $41.1 \pm 0.7$ & $35.8 \pm 0.4$ & $28.5 \pm 0.3$ \\
\hline-2.6 & $100 \pm 1$ & $88.0 \pm 0.6$ & $70.3 \pm 0.4$ \\
\hline-2.2 & $192 \pm 1$ & $168.2 \pm 0.7$ & $135.0 \pm 0.5$ \\
\hline-1.8 & $295 \pm 1$ & $266 \pm 1$ & $215.5 \pm 0.6$ \\
\hline-1.4 & $397 \pm 2$ & $357 \pm 1$ & $293.3 \pm 0.6$ \\
\hline-1.0 & $456 \pm 2$ & $422 \pm 1$ & $348.4 \pm 0.6$ \\
\hline-0.6 & $481 \pm 2$ & $447 \pm 1$ & $380 \pm 9$ \\
\hline-0.2 & $471 \pm 2$ & $449 \pm 2$ & $374.4 \pm 0.7$ \\
\hline 0.2 & $475 \pm 2$ & $448 \pm 2$ & $374.9 \pm 0.7$ \\
\hline 0.6 & $477 \pm 2$ & $445 \pm 2$ & $380 \pm 9$ \\
\hline 1.0 & $457 \pm 2$ & $421 \pm 1$ & $348.8 \pm 0.5$ \\
\hline 1.4 & $396 \pm 2$ & $359 \pm 1$ & $292.8 \pm 0.5$ \\
\hline 1.8 & $294 \pm 1$ & $265 \pm 1$ & $214.9 \pm 0.5$ \\
\hline 2.2 & $191 \pm 2$ & $167.9 \pm 0.6$ & $135.3 \pm 0.5$ \\
\hline 2.6 & $101.1 \pm 0.9$ & $89.0 \pm 0.5$ & $70.1 \pm 0.4$ \\
\hline 3.0 & $41.8 \pm 0.6$ & $36.1 \pm 0.4$ & $28.7 \pm 0.3$ \\
\hline 3.4 & $11.3 \pm 0.3$ & $9.8 \pm 0.2$ & $7.7 \pm 0.2$ \\
\hline 3.8 & $1.5 \pm 0.1$ & $1.16 \pm 0.09$ & $0.92 \pm 0.07$ \\
\hline
\end{tabular}


Table C.7 The transverse-momentum distribution of the hard jet at the LHC

\begin{tabular}{lccc}
\hline$p_{\mathrm{T}, \text { jet }}[\mathrm{GeV}]$ & $\frac{\mathrm{d} \sigma}{\mathrm{d} p_{\mathrm{T}, \text { jet }}}\left[\frac{\mathrm{fb}}{\mathrm{GeV}}\right]$ & & \\
\cline { 2 - 4 } & $\mu=m_{\mathrm{t}} / 2$ & $\mu=m_{\mathrm{t}}$ & $\mu=2 m_{\mathrm{t}}$ \\
\hline 25 & 0 & 0 & 0 \\
75 & $4201 \pm 11$ & $4045 \pm 5$ & $3553 \pm 4$ \\
125 & $1762 \pm 5$ & $1635 \pm 2$ & $1405 \pm 2$ \\
175 & $863 \pm 3$ & $802 \pm 2$ & $685 \pm 1$ \\
225 & $454 \pm 3$ & $428 \pm 1$ & $368.0 \pm 0.8$ \\
275 & $254 \pm 2$ & $242.7 \pm 0.7$ & $210.9 \pm 0.5$ \\
325 & $144 \pm 1$ & $142.1 \pm 0.5$ & $124.9 \pm 0.4$ \\
375 & $85.9 \pm 0.7$ & $86.8 \pm 0.4$ & $76.2 \pm 0.3$ \\
425 & $51.5 \pm 0.5$ & $53.8 \pm 0.3$ & $47.7 \pm 0.2$ \\
475 & $31.3 \pm 0.6$ & $34.1 \pm 0.2$ & $30.6 \pm 0.1$ \\
525 & $19.1 \pm 0.5$ & $21.8 \pm 0.3$ & $20.0 \pm 0.1$ \\
575 & $12.6 \pm 0.2$ & $14.7 \pm 0.2$ & $13.01 \pm 0.08$ \\
625 & $7.4 \pm 0.2$ & $9.4 \pm 0.1$ & $9.06 \pm 0.06$ \\
675 & $4.7 \pm 0.1$ & $6.40 \pm 0.09$ & $6.07 \pm 0.05$ \\
\hline
\end{tabular}

Table C.8 The transverse-momentum distribution of the total $\bar{t} \bar{t}$ system at the LHC

\begin{tabular}{lccc}
\hline$p_{\mathrm{T}, \mathrm{tt}}[\mathrm{GeV}]$ & $\frac{\mathrm{d} \sigma}{\mathrm{d} p_{\mathrm{T}, \mathrm{tt}}}\left[\frac{\mathrm{fb}}{\mathrm{GeV}}\right]$ & & \\
& $\mu=m_{\mathrm{t}} / 2$ & $\mu=m_{\mathrm{t}}$ & $\mu=2 m_{\mathrm{t}}$ \\
\hline 25 & $2390 \pm 10$ & $1457 \pm 6$ & $920 \pm 2$ \\
75 & $1512 \pm 11$ & $2418 \pm 5$ & $2524 \pm 4$ \\
125 & $1915 \pm 5$ & $1722 \pm 3$ & $1460 \pm 1$ \\
175 & $938 \pm 3$ & $843 \pm 2$ & $714 \pm 1$ \\
225 & $496 \pm 2$ & $454 \pm 1$ & $383.9 \pm 0.7$ \\
275 & $274 \pm 1$ & $254.4 \pm 0.7$ & $217.9 \pm 0.5$ \\
325 & $157 \pm 1$ & $148.7 \pm 0.5$ & $128.2 \pm 0.4$ \\
375 & $88 \pm 1$ & $89.2 \pm 0.4$ & $77.7 \pm 0.3$ \\
425 & $54.5 \pm 0.8$ & $54.9 \pm 0.3$ & $48.3 \pm 0.2$ \\
475 & $31.6 \pm 0.6$ & $34.1 \pm 0.2$ & $30.7 \pm 0.2$ \\
525 & $18.8 \pm 0.6$ & $21.4 \pm 0.2$ & $19.8 \pm 0.1$ \\
575 & $11.5 \pm 0.2$ & $13.3 \pm 0.2$ & $13.07 \pm 0.09$ \\
625 & $6.6 \pm 0.2$ & $9.2 \pm 0.2$ & $8.50 \pm 0.08$ \\
675 & $3.7 \pm 0.1$ & $5.91 \pm 0.07$ & $5.72 \pm 0.06$ \\
\hline
\end{tabular}

\section{Appendix C: Tables for histograms}

In this appendix we give the tables for the differential distributions. For each distribution, we list the NLO predictions for the scale choice $\mu=m_{\mathrm{t}} / 2, \mu=m_{\mathrm{t}}$ and $\mu=2 m_{\mathrm{t}}$. In all tables we have set $\mu=\mu_{\text {ren }}=\mu_{\text {fact }}$. The errors result from the Monte Carlo integration. The bin is specified by its central value. The bin width-which we chose constant for the entire histogram - is obtained from the distance of two neighboring bin positions. Note that we use the same definition for the cross section as described in Sect. 3. In partic-
Table C.9 The transverse-momentum distribution of the top quark at the LHC

\begin{tabular}{lccc}
\hline$p_{\mathrm{T}, \mathrm{t}}[\mathrm{GeV}]$ & $\frac{\mathrm{d} \sigma}{\mathrm{d} p_{\mathrm{T}, \mathrm{t}}}\left[\frac{\mathrm{fb}}{\mathrm{GeV}}\right]$ & & \\
& $\mu=m_{\mathrm{t}} / 2$ & $\mu=m_{\mathrm{t}}$ & $\mu=2 m_{\mathrm{t}}$ \\
\hline 25 & $995 \pm 3$ & $881 \pm 2$ & $740 \pm 2$ \\
75 & $2152 \pm 5$ & $1930 \pm 4$ & $1639 \pm 2$ \\
125 & $2029 \pm 6$ & $1858 \pm 3$ & $1597 \pm 3$ \\
175 & $1338 \pm 11$ & $1276 \pm 2$ & $1115 \pm 2$ \\
225 & $723 \pm 7$ & $744 \pm 2$ & $662 \pm 2$ \\
275 & $368 \pm 2$ & $400 \pm 1$ & $365.8 \pm 0.9$ \\
325 & $178 \pm 2$ & $209.3 \pm 0.8$ & $196.4 \pm 0.5$ \\
375 & $83 \pm 1$ & $110.5 \pm 0.6$ & $107.5 \pm 0.6$ \\
425 & $37.9 \pm 0.7$ & $58.4 \pm 0.4$ & $60.1 \pm 0.5$ \\
475 & $14.9 \pm 0.6$ & $31.8 \pm 0.3$ & $33.8 \pm 0.2$ \\
525 & $4.5 \pm 0.4$ & $17.5 \pm 0.2$ & $19.6 \pm 0.1$ \\
575 & $0.1 \pm 0.3$ & $9.6 \pm 0.2$ & $11.4 \pm 0.2$ \\
625 & - & $5.4 \pm 0.1$ & $6.6 \pm 0.1$ \\
675 & - & $3.0 \pm 0.1$ & $4.21 \pm 0.07$ \\
\hline
\end{tabular}

Table C.10 The pseudo-rapidity distribution of the top quark at the LHC

\begin{tabular}{|c|c|c|c|}
\hline \multirow[t]{2}{*}{$\eta_{\mathrm{t}}$} & \multicolumn{3}{|l|}{$\frac{\mathrm{d} \sigma}{\mathrm{d} \eta_{\mathrm{t}}}[\mathrm{pb}]$} \\
\hline & $\mu=m_{\mathrm{t}} / 2$ & $\mu=m_{\mathrm{t}}$ & $\mu=2 m_{\mathrm{t}}$ \\
\hline-5.7 & $0.27 \pm 0.04$ & $0.21 \pm 0.01$ & $0.184 \pm 0.008$ \\
\hline-5.1 & $0.85 \pm 0.04$ & $0.71 \pm 0.03$ & $0.58 \pm 0.01$ \\
\hline-4.5 & $2.58 \pm 0.07$ & $2.27 \pm 0.03$ & $1.92 \pm 0.04$ \\
\hline-3.9 & $7.5 \pm 0.1$ & $6.55 \pm 0.05$ & $5.35 \pm 0.05$ \\
\hline-3.3 & $18.3 \pm 0.1$ & $16.3 \pm 0.1$ & $13.54 \pm 0.07$ \\
\hline-2.7 & $36.4 \pm 0.2$ & $32.8 \pm 0.1$ & $27.86 \pm 0.07$ \\
\hline-2.1 & $56.1 \pm 0.2$ & $52.2 \pm 0.1$ & $45.05 \pm 0.09$ \\
\hline-1.5 & $69.6 \pm 0.2$ & $66.7 \pm 0.2$ & $58.3 \pm 0.1$ \\
\hline-0.9 & $70.4 \pm 0.5$ & $69.7 \pm 0.1$ & $61.64 \pm 0.09$ \\
\hline-0.3 & $66.6 \pm 0.2$ & $66.7 \pm 0.1$ & $59.28 \pm 0.09$ \\
\hline 0.3 & $67.0 \pm 0.2$ & $66.5 \pm 0.1$ & $59.39 \pm 0.09$ \\
\hline 0.9 & $71.4 \pm 0.3$ & $69.5 \pm 0.3$ & $61.57 \pm 0.09$ \\
\hline 1.5 & $69.6 \pm 0.2$ & $66.6 \pm 0.3$ & $58.1 \pm 0.1$ \\
\hline 2.1 & $56.3 \pm 0.3$ & $52.8 \pm 0.3$ & $45.10 \pm 0.08$ \\
\hline 2.7 & $36.0 \pm 0.2$ & $32.6 \pm 0.2$ & $27.80 \pm 0.08$ \\
\hline 3.3 & $18.3 \pm 0.1$ & $16.3 \pm 0.1$ & $13.43 \pm 0.08$ \\
\hline 3.9 & $7.8 \pm 0.2$ & $6.57 \pm 0.05$ & $5.43 \pm 0.04$ \\
\hline 4.5 & $2.6 \pm 0.1$ & $2.28 \pm 0.04$ & $1.82 \pm 0.03$ \\
\hline 5.1 & $0.83 \pm 0.04$ & $0.70 \pm 0.02$ & $0.61 \pm 0.01$ \\
\hline 5.7 & $0.25 \pm 0.02$ & $0.20 \pm 0.01$ & $0.171 \pm 0.009$ \\
\hline
\end{tabular}


Table C.11 The rapidity distribution of the top quark at the LHC

\begin{tabular}{lccc}
\hline$y_{\mathrm{t}}$ & $\frac{\mathrm{d} \sigma}{\mathrm{d} y_{\mathrm{t}}}[\mathrm{pb}]$ & & \\
& $\mu=m_{\mathrm{t}} / 2$ & $\mu=m_{\mathrm{t}}$ & $\mu=2 m_{\mathrm{t}}$ \\
\hline-3.9 & $0.093 \pm 0.008$ & $0.075 \pm 0.006$ & $0.053 \pm 0.004$ \\
-3.3 & $2.39 \pm 0.06$ & $1.97 \pm 0.04$ & $1.55 \pm 0.02$ \\
-2.7 & $12.9 \pm 0.1$ & $11.55 \pm 0.09$ & $9.52 \pm 0.05$ \\
-2.1 & $35.7 \pm 0.2$ & $33.3 \pm 0.1$ & $28.35 \pm 0.07$ \\
-1.5 & $66.7 \pm 0.2$ & $63.3 \pm 0.2$ & $55.1 \pm 0.1$ \\
-0.9 & $96.5 \pm 0.3$ & $92.9 \pm 0.2$ & $81.5 \pm 0.1$ \\
-0.3 & $114.1 \pm 0.6$ & $111.1 \pm 0.2$ & $97.7 \pm 0.1$ \\
0.3 & $115.6 \pm 0.3$ & $110.9 \pm 0.2$ & $97.7 \pm 0.1$ \\
0.9 & $96.5 \pm 0.2$ & $92.9 \pm 0.2$ & $81.4 \pm 0.2$ \\
1.5 & $67.0 \pm 0.3$ & $63.5 \pm 0.2$ & $55.0 \pm 0.1$ \\
2.1 & $35.6 \pm 0.2$ & $33.1 \pm 0.1$ & $28.32 \pm 0.07$ \\
2.7 & $12.8 \pm 0.1$ & $11.45 \pm 0.07$ & $9.50 \pm 0.06$ \\
3.3 & $2.32 \pm 0.08$ & $1.95 \pm 0.03$ & $1.56 \pm 0.02$ \\
3.9 & $0.095 \pm 0.006$ & $0.071 \pm 0.005$ & $0.050 \pm 0.003$ \\
\hline
\end{tabular}

Table C.12 The rapidity distribution of the hard jet at the LHC

\begin{tabular}{lccc}
\hline$y_{\text {jet }}$ & $\frac{\mathrm{d} \sigma}{\mathrm{d} y_{\text {jet }}}[\mathrm{pb}]$ & & \\
\cline { 2 - 4 } & $\mu=m_{\mathrm{t}} / 2$ & $\mu=m_{\mathrm{t}}$ & $\mu=2 m_{\mathrm{t}}$ \\
\hline-5.1 & $0.05 \pm 0.04$ & $0.18 \pm 0.02$ & $0.12 \pm 0.01$ \\
-4.5 & $2.2 \pm 0.1$ & $2.09 \pm 0.07$ & $1.88 \pm 0.04$ \\
-3.9 & $9.8 \pm 0.2$ & $9.8 \pm 0.1$ & $8.34 \pm 0.07$ \\
-3.3 & $23.0 \pm 0.2$ & $22.2 \pm 0.1$ & $19.38 \pm 0.08$ \\
-2.7 & $39.1 \pm 0.3$ & $37.1 \pm 0.1$ & $32.21 \pm 0.08$ \\
-2.1 & $53.3 \pm 0.5$ & $50.5 \pm 0.1$ & $44.01 \pm 0.09$ \\
-1.5 & $62.6 \pm 0.5$ & $59.9 \pm 0.2$ & $52.2 \pm 0.1$ \\
-0.9 & $68.3 \pm 0.6$ & $65.1 \pm 0.2$ & $56.8 \pm 0.1$ \\
-0.3 & $71.1 \pm 0.3$ & $67.2 \pm 0.1$ & $58.8 \pm 0.1$ \\
0.3 & $70.7 \pm 0.3$ & $67.0 \pm 0.3$ & $58.8 \pm 0.1$ \\
0.9 & $68.1 \pm 0.3$ & $65.4 \pm 0.3$ & $56.8 \pm 0.2$ \\
1.5 & $63.0 \pm 0.3$ & $60.1 \pm 0.1$ & $52.1 \pm 0.1$ \\
2.1 & $53.6 \pm 0.3$ & $50.5 \pm 0.1$ & $44.0 \pm 0.1$ \\
2.7 & $39.1 \pm 0.3$ & $36.9 \pm 0.1$ & $32.18 \pm 0.09$ \\
3.3 & $22.8 \pm 0.2$ & $22.3 \pm 0.1$ & $19.3 \pm 0.1$ \\
3.9 & $9.9 \pm 0.1$ & $10.2 \pm 0.4$ & $8.34 \pm 0.06$ \\
4.5 & $2.09 \pm 0.09$ & $2.2 \pm 0.1$ & $1.88 \pm 0.06$ \\
5.1 & $0.12 \pm 0.02$ & $0.13 \pm 0.02$ & $0.12 \pm 0.01$ \\
\hline & & &
\end{tabular}

ular, we demand a minimum $p_{\mathrm{T}}$ for the additional jet. For the Tevatron $20 \mathrm{GeV}$ is used, while for the LHC $50 \mathrm{GeV}$ is used.

\section{References}

1. CDF-Run II and D0-Run II, U. Heintz (2008). arXiv: 0806.1202

2. W. Bernreuther, J. Phys. G 35, 083001 (2008). arXiv:0805.1333
3. T. Han (2008). arXiv:0804.3178

4. E. Vanes, Plenary Talk at ICHEP 08. Philadelphia, USA, Jul. 29th-Aug. 5th, 2008

5. D0, V.M. Abazov et al., Phys. Rev. Lett. 98, 181802 (2007). hep-ex/0612052

6. D0, V.M. Abazov et al., Phys. Rev. D 78, 012005 (2008). arXiv: 0803.0739

7. CDF, T. Aaltonen et al. (2008). arXiv:0809.2581

8. B.W. Harris, E. Laenen, L. Phaf, Z. Sullivan, S. Weinzierl, Phys. Rev. D 66, 054024 (2002). hep-ph/0207055

9. Z. Sullivan, Phys. Rev. D 70, 114012 (2004). hep-ph/0408049

10. N. Kidonakis, Phys. Rev. D 74, 114012 (2006). hep-ph/0609287

11. N. Kidonakis, Phys. Rev. D 75, 071501 (2007). hep-ph/0701080

12. CDF, A.A. Affolder et al., Phys. Rev. Lett. 84, 216 (2000). hep-ex/ 9909042

13. CDF, D.E. Acosta et al., Phys. Rev. D 71, 031101 (2005). hep-ex/ 0411070

14. CDF, A. Abulencia et al., Phys. Rev. D 73, 111103 (2006). hep-ex/ 0511023

15. CDF II, A. Abulencia et al., Phys. Rev. D 75, 052001 (2007). hep-ex/0612011

16. D0, V.M. Abazov et al., Phys. Rev. Lett. 100, 062004 (2008). arXiv:0711.0032

17. D0, V.M. Abazov et al., Phys. Rev. D 75, 031102 (2007). hep-ex/ 0609045

18. D0, V.M. Abazov et al., Phys. Lett. B 617, 1 (2005). hep-ex/ 0404040

19. W. Bernreuther, A. Brandenburg, Z.G. Si, P. Uwer, Phys. Rev. Lett. 87, 242002 (2001). hep-ph/0107086

20. W. Bernreuther, A. Brandenburg, Z.G. Si, P. Uwer, Nucl. Phys. B 690, 81 (2004). hep-ph/0403035

21. A. Lazopoulos, T. McElmurry, K. Melnikov, F. Petriello (2008). arXiv:0804.2220

22. W. Beenakker et al., Phys. Rev. Lett. 87, 201805 (2001). hep-ph/ 0107081

23. W. Beenakker et al., Nucl. Phys. B 653, 151 (2003). hep-ph/ 0211352

24. S. Dawson, L.H. Orr, L. Reina, D. Wackeroth, Phys. Rev. D 67, 071503 (2003). hep-ph/0211438

25. S. Dawson, C. Jackson, L.H. Orr, L. Reina, D. Wackeroth, Phys. Rev. D 68, 034022 (2003). hep-ph/0305087

26. S. Moch, P. Uwer (2008). arXiv:0804.1476

27. F. Halzen, P. Hoyer, C.S. Kim, Phys. Lett. B 195, 74 (1987)

28. J.H. Kühn, G. Rodrigo, Phys. Rev. D 59, 054017 (1999). hep-ph/ 9807420

29. J.H. Kühn, G. Rodrigo, Phys. Rev. Lett. 81, 49 (1998). hep-ph/ 9802268

30. M.T. Bowen, S.D. Ellis, D. Rainwater, Phys. Rev. D 73, 014008 (2006). hep-ph/0509267

31. F.A. Berends, K.J.F. Gaemers, R. Gastmans, Nucl. Phys. B 63, 381 (1973)

32. F.A. Berends, R. Kleiss, S. Jadach, Z. Was, Acta Phys. Pol. B 14, 413 (1983)

33. D0, V.M. Abazov et al., Phys. Rev. Lett. 100, 142002 (2008). arXiv:0712.0851

34. CDF, T. Aaltonen et al. (2008). arXiv:0806.2472

35. O. Antunano, J.H. Kühn, G. Rodrigo, Phys. Rev. D 77, 014003 (2008). arXiv:0709.1652

36. L.G. Almeida, G. Sterman, W. Vogelsang, Phys. Rev. D 78, 014008 (2008). arXiv:0805.1885

37. S. Dittmaier, P. Uwer, S. Weinzierl, Phys. Rev. Lett. 98, 262002 (2007). hep-ph/0703120

38. M. Cacciari, S. Frixione, M.M. Mangano, P. Nason, G. Ridolfi (2008). arXiv:0804.2800

39. N. Kidonakis, R. Vogt (2008). arXiv:0805.3844

40. J.G. Körner, Z. Merebashvili, M. Rogal, Phys. Rev. D 73, 034030 (2006). hep-ph/0511264 
41. J.G. Körner, Z. Merebashvili, M. Rogal, Phys. Rev. D 77, 094011 (2008). arXiv:0802.0106

42. S. Moch, A. Mitov, Acta Phys. Pol. B 38, 3507 (2007). arXiv: 0711.1121

43. M. Czakon, A. Mitov, S. Moch, Phys. Lett. B 651, 147 (2007). arXiv:0705.1975

44. M. Czakon, A. Mitov, S. Moch, Nucl. Phys. B 798, 210 (2008). arXiv:0707.4139

45. M. Czakon, Phys. Lett. B 664, 307 (2008). arXiv:0803.1400

46. R. Bonciani, A. Ferroglia, T. Gehrmann, D. Maitre, C. Studerus (2008). arXiv:0806.2301

47. C. Anastasiou, S.M. Aybat (2008). arXiv:0809.1355

48. B. Kniehl, J.G. Korner, Z. Merebashvili, M. Rogal (2008). arXiv: 0809.3980

49. R.K. Ellis, W.T. Giele, Z. Kunszt, K. Melnikov (2008). arXiv: 0806.3467

50. S. Asai et al., Eur. Phys. J. C 32S2, 19 (2004). hep-ph/0402254

51. S. Abdullin et al., Eur. Phys. J. C 39S2, 41 (2005)

52. A. Alves, O. Eboli, T. Plehn, D.L. Rainwater, Phys. Rev. D 69, 075005 (2004). hep-ph/0309042

53. C. Buttar et al. (2006). hep-ph/0604120

54. NLO Multileg Working Group, Z. Bern, et al. (2008). arXiv:0803. 0494

55. T. Stelzer, W.F. Long, Comput. Phys. Commun. 81, 357 (1994). hep-ph/9401258

56. J. Küblbeck, M. Böhm, A. Denner, Comput. Phys. Commun. 60, 165 (1990)

57. T. Hahn, Comput. Phys. Commun. 140, 418 (2001). hep-ph/ 0012260

58. S. Dittmaier, Nucl. Phys. B 675, 447 (2003). hep-ph/0308246

59. A. Bredenstein, A. Denner, S. Dittmaier, S. Pozzorini, J. High Energy Phys. 08, 108 (2008). arXiv:0807.1248

60. A. Denner, S. Dittmaier, Nucl. Phys. B 658, 175 (2003). hep-ph/ 0212259

61. T. Binoth, J.P. Guillet, G. Heinrich, E. Pilon, C. Schubert, J. High Energy Phys. 10, 015 (2005). hep-ph/0504267

62. G. Passarino, M.J.G. Veltman, Nucl. Phys. B 160, 151 (1979)
63. G. 't Hooft, M.J.G. Veltman, Nucl. Phys. B 153, 365 (1979)

64. W. Beenakker, A. Denner, Nucl. Phys. B 338, 349 (1990)

65. A. Denner, U. Nierste, R. Scharf, Nucl. Phys. B 367, 637 (1991)

66. A. Denner, S. Dittmaier, Nucl. Phys. B 734, 62 (2006). hep-ph/ 0509141

67. P. Nogueira, J. Comput. Phys. 105, 279 (1993)

68. J.A.M. Vermaseren (2000). math-ph/0010025

69. W.T. Giele, E.W.N. Glover, J. High Energy Phys. 04, 029 (2004). hep-ph/0402152

70. G.J. van Oldenborgh, J.A.M. Vermaseren, Z. Phys. C 46, 425 (1990)

71. G.J. van Oldenborgh, Comput. Phys. Commun. 66, 1 (1991)

72. W. Giele, E.W.N. Glover, G. Zanderighi, Nucl. Phys. Proc. Suppl. 135, 275 (2004). hep-ph/0407016

73. S. Catani, M.H. Seymour, Nucl. Phys. B 485, 291 (1997). hep-ph/ 9605323

74. L. Phaf, S. Weinzierl, J. High Energy Phys. 04, 006 (2001). hep-ph/0102207

75. S. Catani, S. Dittmaier, M.H. Seymour, Z. Trócsányi, Nucl. Phys. B 627, 189 (2002). hep-ph/0201036

76. S. Weinzierl, Eur. Phys. J. C 45, 745 (2006). hep-ph/0510157

77. F.A. Berends, W.T. Giele, Nucl. Phys. B 306, 759 (1988)

78. S. Weinzierl, D.A. Kosower, Phys. Rev. D 60, 054028 (1999). hep-ph/9901277

79. J. Pumplin et al., J. High Energy Phys. 07, 012 (2002). hep-ph/ 0201195

80. S.D. Ellis, D.E. Soper, Phys. Rev. D 48, 3160 (1993). hep-ph/ 9305266

81. S. Frixione, P. Nason, B.R. Webber, J. High Energy Phys. 08, 007 (2003). hep-ph/0305252

82. M.L. Mangano, M. Moretti, F. Piccinini, M. Treccani, J. High Energy Phys. 01, 013 (2007). hep-ph/0611129

83. T. Gleisberg, F. Krauss, Eur. Phys. J. C 53, 501 (2008). arXiv: 0709.2881

84. M.H. Seymour, C. Tevlin (2008). arXiv:0803.2231

85. K. Hasegawa, S. Moch, P. Uwer (2008). arXiv:0807.3701

86. R. Frederix, T. Gehrmann, N. Greiner (2008). arXiv:0808.2128 